Al1106 339944

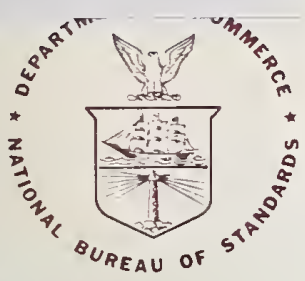

NBS TECHNICAL NOTE 1054

REFERENGE

NBS

PUBLICATIONS

U.S. DEPARTMENT OF COMMERCE / National Bureau of Standards

\title{
Input Impedance of a Probe Antenna Exciting a TEM Cell
}

$Q C$
100
.$U 5753$
$\# 1054$
1982




\section{NATIONAL BUREAU OF STANDARDS}

The National Bureau of Standards' was established by an act of Congress on March 3, 1901. The Bureau's overall goal is to strengthen and advance the Nation's science and technology and facilitate their effective application for public benefit. To this end, the Bureau conducts research and provides: (1) a basis for the Nation's physical measurement system, (2) scientific and technological services for industry and government, (3) a technical basis for equity in trade, and (4) technical services to promote public safety. The Bureau's technical work is performed by the National Measurement Laboratory, the National Engineering Laboratory, and the Institute for Computer Sciences and Technology.

THE NATIONAL MEASUREMENT LABORATORY provides the national system of physical and chemical and materials measurement; coordinates the system with measurement systems of other nations and furnishes essential services leading to accurate and uniform physical and chemical measurement throughout the Nation's scientific community, industry, and commerce; conducts materials research leading to improved methods of measurement, standards, and data on the properties of materials needed by industry, commerce, educational institutions, and Government; provides advisory and research services to other Government agencies; develops, produces, and distributes Standard Reference Materials; and provides calibration services. The Laboratory consists of the following centers:

\section{Absolute Physical Quantities ${ }^{2}$ - Radiation Research - Chemical Physics - Analytical Chemistry - Materials Science}

THE NATIONAL ENGINEERING LABORATORY provides technology and technical services to the public and private sectors to address national needs and to solve national problems; conducts research in engineering and applied science in support of these efforts; builds and maintains competence in the necessary disciplines required to carry out this research and technical service; develops engineering data and measurement capabilities; provides engineering measurement traceability services; develops test methods and proposes engineering standards and code changes; develops and proposes new engineering practices; and develops and improves mechanisms to transfer results of its research to the ultimate user. The Laboratory consists of the following centers:

Applied Mathematics - Electronics and Electrical Engineering ${ }^{2}$ - Manufacturing Engineering - Building Technology - Fire Research - Chemical Engineering ${ }^{2}$

THE INSTITUTE FOR COMPUTER SCIENCES AND TECHNOLOGY conducts research and provides scientific and technical services to aid Federal agencies in the selection, acquisition, application, and use of computer technology to improve effectiveness and economy in Government operations in accordance with Public Law 89-306 (40 U.S.C. 759), relevant Executive Orders, and other directives; carries out this mission by managing the Federal Information Processing Standards Program, developing Federal ADP standards guidelines, and managing Federal participation in ADP voluntary standardization activities; provides scientific and technological advisory services and assistance to Federal agencies; and provides the technical foundation for computer-related policies of the Federal Government. The Institute consists of the following centers:

Programming Science and Technology - Computer Systems Engineering.

'Headquarters and Laboratories at Gaithersburg, MD, unless otherwise noted; mailing address Washington, DC 20234.

${ }^{2}$ Some divisions within the center are located at Boulder, CO 80303. 


\title{
Input Impedance of a Probe Antenna Exciting a TEM Cell
}

\author{
P. F. Wilson $\dagger$ \\ D. C. Chang $\dagger$ \\ M. T. Ma
}

Electromagnetic Fields Division

National Engineering Laboratory

National Bureau of Standards

Boulder, Colorado 80303

†Department of Electrical Engineering

University of Colorado

Boulder, Colorado 80309

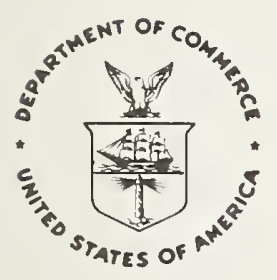

U.S. DEPARTMENT OF COMMERCE, Malcolm Baldrige, Secretary

NATIONAL BUREAU OF STANDARDS, Ernest Ambler, Director 
NATIONAL BUREAU OF STANDARDS TECHNICAL NOTE 1054 Nat. Bur. Stand. (U.S.), Tech. Note 1054, 52 pages (April 1982) CODEN: NBTNAE 
FOREWORD iv

1. INTRODUCTION. .1

2. VARIATIONAL FORMULA FOR THE PROBE INPUT IMPEDANCE............

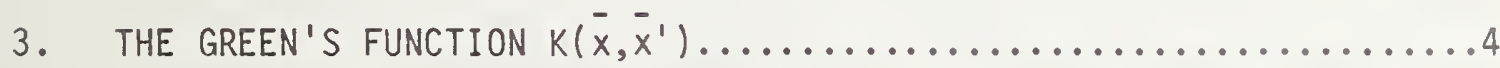

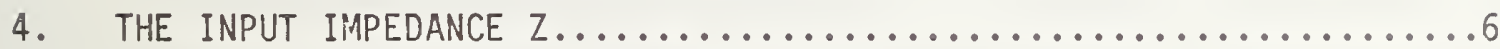

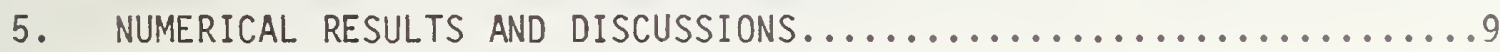

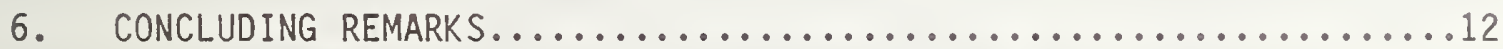

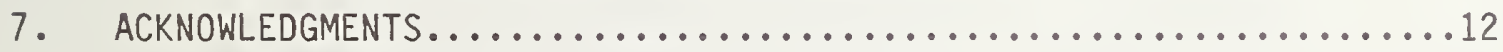

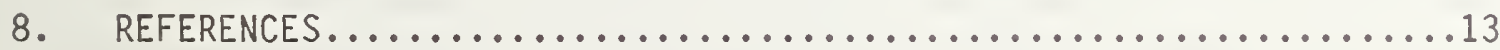

APPENDIX A. Derivation of $\tilde{K}_{1}^{(j)}$ and $\tilde{K}_{2}^{(j)} \ldots \ldots \ldots \ldots \ldots$

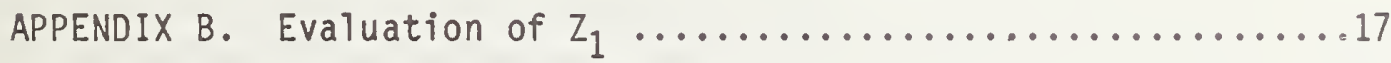

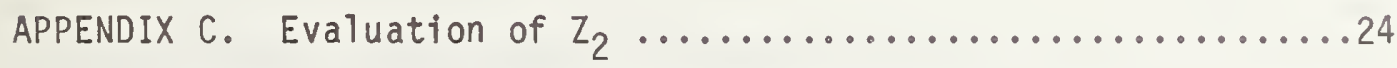

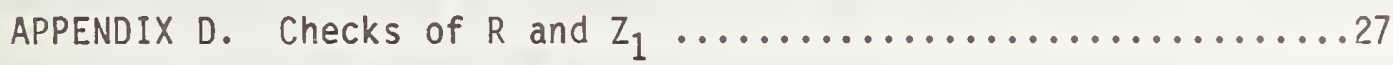

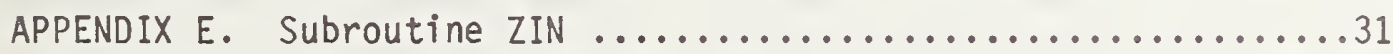

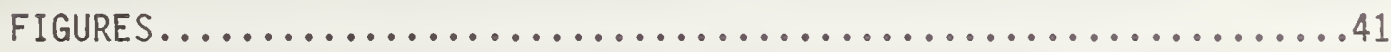


FOREWORD

This report describes theoretical and experimental analyses developed by staff of the University of Colorado at Boulder in collaboration with the Electromagnetic Fields Division of the National Bureau of Standards (NBS), under a contract sponsored by NBS. Professor David C. Chang heads the University team. Dr. Mark T. Ma of NBS serves as the technical contract monitor. The period covered by this report extends from July 1980 to July 1981.

The work presented in this report represents a further aspect of establishing a theoretical basis for the technical analyses of transverse electromagnetic (TEM) transmission line cells. The general purpose of pursuing theoretical studies is to evaluate the use of TEM cells for (1) measuring the total of radiated power by a device inserted into the cell for test, or (2) performing necessary susceptibility tests on a small electronic device.

The particular topic addressed herein is to determine the input impedance of a probe antenna which may be inserted into a TEM cell to measure or excite fields. The formulation of the problem is based on a variational principle, which insures that the input impedance so obtained is stationary for small arbitrary variations in the probe current distribution about its true value. The evaluation of the variational integral requires a knowledge of the field distribution inside the cell excited by a vertical short electric dipole. This latter problem was the subject of a previous report by the same authors [4], the results of which are readily adapted to the present problem. Thus, the input impedance treated in this report represents an extension to the previous studies.

The analysis of the present problem is significantly simplified by assuming that the gap between the inner septum and the outer walls of the cell is electrically small, and that the operating frequency is such that no higher order modes are excited inside the cell. These assumptions are consistent with the practical cell utilization and design principle.

The final impedance expression is shown to consist of two distinct terms, which can be clearly identified as the respective contributions by the ordinary rectangular waveguide and the gap perturbation. 
Previous publications under the same effort include:

Tippet, J. C. and Chang, D. C., Radiation characteristics of dipole sources located inside a rectangular coaxial transmission line, NBSIR 75-829 (Jan. 1976).

Tippet, J. C., Chang, D. C., and Crawford, M. L., An analytical and experimental determination of the cut-off frequencies of higher-order TE modes in a TEM cell, NBSIR 76-841 (June 1976).

Tippet, J. C. and Chang, D. C., Higher-order modes in rectangular coaxial line with infinitely thin inner conductor, NBSIR 78-873 (March 1978).

Sreenivasiah, I. and Chang, D. C., A variational expression for the scattering matrix of a coaxial line step discontinuity and its application to an over moded coaxial TEM cell, NBSIR 79-1606 (May 1979).

Tippet, J. C. and Chang, D. C., Dispersion and attenuation characteristics of modes in a TEM cell with a lossy dielectric slab, NBSIR 79-1615 (Aug. 1979).

Sreenivasiah, I., Chang, D. C., and Ma, M. T., Characterization of electrically small radiating sources by tests inside a transmission line cell, NBS Tech Note 1017 (Feb. 1980).

Wilson, P. F., Chang, D. C., and Ma, M. T., Excitation of a TEM cell by a vertical electric Hertzian dipole, NBS Tech Note 1037 (March 1981).

Sreenivasiah, I., Chang, D. C., and Ma, M. T., A method of determinina the emission and susceptibility levels of electrically small objects using a TEM cell, NBS Tech Note 1040 (April 1981). 



\title{
INPUT IMPEDANCE OF A PROBE ANTENNA EXCITING A TEM CELL
}

\author{
P. F. Wilson, D. C. Chang and M. T. Ma
}

The input impedance of a probe antenna exciting a transverse electromagnetic (TEM) transmission line cell is formulated by a variational approach. The formulation also utilizes the results from a previous work on the field distribution inside a TEM cell excited by a vertical electrical Hertzian dipole. The final result of impedance is shown to consist of two distinct terms, which are respectively contributed by the ordinary rectangular waveguide and the gap perturbation. Numerical results for both the real and imaginary parts of the impedance are given. The resistive part is found to be proportional to the square of the probe length, and the reactive part largely capacitive.

Key words: Green's function; input impedance; probe antenna; radiation resistance; rectangular coaxial transmission line; TEM cel1; variational method.

\section{INTRODUCTION}

The National Bureau of Standards (NBS) has been interested in developing a method for testing the emission and susceptibility properties of electronic equipment. One approach to this problem is the transverse electromagnetic (TEM) transmission line cell. The TEM cell, shown in figure 1, consists of a section of rectangular coaxial tramission line (RCTL) coupled at each end to standard $50 \Omega$ cylindrical coaxial line via a tapered section. The RCTL section supports a dominant TEM mode and provides an isolated, standard test field environment. The TEM cell results may be related to the free space environment via results given by Tippet [1] and Sreenivasiah [2].

This report concerns the manner in which an idealized coaxial probe excites an infinite section of RCTL. The results may be used to study the effect of coaxial probes inserted into the TEM cell either to excite, or to measure fields. In addition, the configuration studied may be useful in modeling feed lines to electronic equipment. The method of solution is to formulate a variational expression for the input impedance of the probe at the aperture plane, as was done by collin [3] in analyzing the coaxial probe excitation of a rectangular waveguide. The evaluation of the variational integral requires that the Green's function corresponding to the fields excited in an RCTL by a vertical electric dipole (VED) be known. The VED excitation of an RCTL was the subject of a previous report by the authors [4], 
the results of which will be adapted to the present problem. The analysis of the Green's function is significantly simplified by assuning that the gap between the inner septum and the outer wal1s, is electrically small, and that the operating frequency is not too large. Both these premises are consistent with the TEM cell usage.

Evaluating the variational impedance integral results in two physically distinct terms, which may be referred to as the ordinary rectangular waveguide contribution, and the gap perturbation contribution. The ordinary term gives the input reactance due to a coaxial probe in a rectangular waveguide with the same dimensions as that of the RCTL's upper chamber. Since the dimensions of the cell are such that the rectangular waveguide modes in the upper chamber would be cutoff, the result is a highly capacitive term for which the analysis yields an approximate analytic expression. The gap perturbation term represents the change from the ordinary result due to the presence of the gap fields, and contains the radiation resistance resulting from the dominant TEM mode. The perturbation term, as derived, remains in an integral form which must be treated numerically. However, if the TEM mode radiation resistance is extracted, the remaining reactive integral is negligible when compared to the highly capacitive ordinary contribution. Thus neglecting the perturbation reactance results in a computationally simple algebraic form for the probe input impedance.

Section 2 of this report will define the variational impedance integral to be evaluated and discuss the necessary probe idealizations. The Green's function associated with the VED excitation of an RCTL is examined in Section 3. Section 4 gives the result of the evaluation of the impedance integral and examines the distinct physical nature of the resulting terms. Certain special cases are used to verify the solution. These checks are examined in Section 5 along with numerical results. Many details of the analysis and a listing of the FORTRAN program written to evaluate the impedance terms are left to the Appendices. 


\section{VARIATIONAL FORMULA FOR THE PROBE INPUT IMPEDANCE}

The RCTL cross section and the coaxial-line probe antenna are depicted in figure 2. The septum, of width $2 w$, is allowed to be vertically offset with the heights of the upper and lower chambers given by $b_{1}$, and $b_{2}$, respectively. The width of the upper chamber is $2 a_{1}$, and the width of the lower chamber is $2 \mathrm{a}_{2}$. The gap between the septum and the outer wall is $\mathrm{g}$. The probe, of length $d$ and radius $t$, is located in the upper chamber. A TEM cell would have an RCTL cross section with equal chamber widths and previous discussions have assumed this geometry. However, in order to avoid any degeneracies caused by letting $a_{1}=a_{2}=a$, the analysis will initially assume general chamber widths. The time convention will be $\exp (-i \omega t)$.

If the coaxial-line aperture is small, it is reasonable to neglect the higher order modes excited at the aperture discontinuity and assume only incident and reflected TEM modes exist in the coaxial-line. As shown by Collin [3] in his analysis of the coaxial-line probe antenna excitation of an ordinary rectangular waveguide, the above approximation allows the antenna input impedance $Z$ to be expressed as follows

$$
Z=\frac{-1}{I_{t}^{2}} \int_{S} \int_{S^{\prime}} \bar{J}(\bar{x}) \cdot \overline{\bar{k}}\left(\bar{x}, \bar{x}^{\prime}\right) \cdot \bar{J}\left(\bar{x}^{\prime}\right) d s^{\prime} d s
$$

where $\bar{x}$ and $\bar{x}^{\prime}$ denote source and observation points, $\bar{j}(\bar{x})$ is the unknown current density on the probe, $\overline{\bar{k}}\left(\bar{x}, \bar{x}^{\prime}\right)$ is a dyadic Green's function corresponding to the electric field excited by a unit strength dipole source, and $I_{t}$ is the total current at the aperture plane. S denotes the probe surface plus the aperture plane which carries an equivalent electric current $\bar{J}(\bar{x})=\bar{n} \times \bar{H}(\bar{x})$ where $\bar{n}$ is a unit vector in the outward normal direction. Experimental studies have shown that $z$ is effectively independent of the aperture size for electrically small apertures [3]. Therefore it is reasonable to idealize the probe as driven by a y-directed E-field concentrated at the base. Thus we need consider a $y$-directed current only, which reduces $\bar{J}(\bar{x})$ to $\bar{a}_{y} J(\bar{x})$, and $\overline{\bar{k}}\left(\bar{x}, \bar{x}^{\prime}\right)$ to $\bar{a}_{y} k\left(\bar{x}, \bar{x}^{\prime}\right) \bar{a}_{y}$ with $\bar{a}_{y}$ being the y-directed unit vector. 
The above impedance expression may be shown to be variational in $J(\bar{x})$. That is, a first order approximation for $J(\bar{x})$ leads to a second order approximation for $Z$. At normal TEM cell operating frequencies, the length of the probe will be small compared to a wavelength. Therefore, it is reasonable to assume that the current distribution is approximately sinusoidal,

$$
J(\bar{x})=\frac{I_{0}}{2 \pi t} \sin k\left(b_{1}-d-y\right) \delta(\rho-t)
$$

where the delta function is interpreted in the sense of a generalized function. The form of the Green's function $K\left(\bar{x}, \bar{x}^{\prime}\right)$ will be considered next.

\section{THE GREEN'S FUNCTION $K\left(\bar{x}, \overline{x^{\prime}}\right)$}

The appropriate Green's function for the special case of equal chamber widths has been derived previously [4]. However, the modifications due to generalizing the chamber widths are not difficult. Since a VED in an RCTL will excite both transverse electric (TE or h-type), and transverse magnetic (TM or e-type) modes, both cases need to be treated. For each modal type we begin by formulating an integral equation for the unknown field in the gap. These integral equations could be solved by Chebyshev polynomial expansion methods [5]. However, a less cumbersome solution may be achieved by making the following pair of assumptions. First, the gap is assumed to be small, and second, the frequency is not allowed to be too high. These assumptions lead to an approximate integral equation which may be solved directly. The small gap assumption amounts to discarding terms which are of the order $G^{2} \ell n G$, where $G$ is a normalized gap parameter defined by $G=\pi g / 2 a, a=m i n\left(a_{1}, a_{2}\right)$. Up to the order of this approximation only the TE type modes contribute significantly to the gap field.

The derivation of the Green's function begins with the removal of the zdependence via a Fourier-transform defined by

$$
k^{(j)}\left(\bar{x}, \bar{x}^{\prime}\right)=\frac{1}{2 \pi} \int_{-\infty}^{\infty} \tilde{k}^{(j)}\left(\bar{x}_{t}, \bar{x}_{t}^{\prime}\right) e^{i \alpha z} d \alpha
$$


where $j=1,2$ refer to the upper, and lower chambers respectively, the subscript $t$ denotes the transverse $p l a n e, \alpha$ is the transform variable representing a continuum of propagation factors, and the superscript tilde signifies a function in the transform domain. The analysis outlined above yields the following result

$$
\tilde{k}^{(j)}\left(\bar{x}_{t}, \bar{x}_{t}^{\prime}\right)=\tilde{k}_{1}^{(j)}\left(\bar{x}_{t}, \bar{x}_{t}^{\prime}\right)+\tilde{k}_{2}^{(j)}\left(\bar{x}_{t}, \bar{x}_{t}^{\prime}\right)
$$

where $\tilde{k}_{1}^{(j)}$ represents the Green's function expected if there were no gap, i.e., the ordinary rectangular waveguide result, and $\tilde{K}_{2}^{(j)}$ gives the gap field perturbation contribution. $\tilde{K}_{1}^{(j)}$ and $\tilde{K}_{2}^{(j)}$ derived in Appendix $A$ are found to be

$$
\begin{gathered}
\tilde{k}_{1}^{(j)}\left(\bar{x}_{t}, \bar{x}_{t}^{\prime}\right)=-\delta_{1 j} \frac{i \omega \mu}{k^{2}}\left\{\frac{\alpha^{2}}{\zeta} \delta\left(\bar{x}_{t}-\bar{x}_{t}^{\prime}\right)+\sum_{m, n=0}^{\infty} \frac{\Delta_{n}\left[M_{j}^{2}+\alpha^{2}\right]}{m_{j}^{2}+N_{j}^{2}-\zeta^{2}} g_{m n}^{(j)}\left(\bar{x}_{t}, \bar{x}_{t}^{\prime}\right)\right\} \\
\tilde{k}_{2}^{(j)}\left(\bar{x}_{t}, \bar{x}_{t}^{\prime}\right)=\frac{i \omega \mu \pi}{2 a_{1} a_{j} \zeta^{2}} L(\alpha) f_{j}\left(\bar{x}_{t}\right) f_{1}\left(\bar{x}_{t}^{\prime}\right)
\end{gathered}
$$

where $\omega=2 \pi f$ is the angular frequency, $\mu$ is the permeability, $k=\omega(\mu \varepsilon)^{1 / 2}$ is the TEM mode propagation constant, $\delta_{i j}$ is the Kronecker delta function, $\zeta^{2}=$ $k^{2}-\alpha^{2}, M_{j}=m \pi / 2 a_{j}$, and $i_{j}=n \pi / b_{j}$. The remaining functions are defined by

$$
\begin{aligned}
& g_{m n}^{(j)}\left(\bar{x}_{t}, \bar{x}_{t}^{\prime}\right)=\left(\frac{2}{a_{j} b_{j}}\right) \operatorname{sinim}\left(x+a_{j}\right) \sin M_{j}\left(x^{\prime}+a_{j}\right) \cos N_{j} y \cos N_{j} y^{\prime} \\
& L(\alpha)=\left\{\ln \frac{8 \sqrt{a_{1} a_{2}}}{\pi g}+\frac{\pi}{2} \sum_{j=1}^{2} \frac{1}{a_{j}} \sum_{m_{0}} \frac{\operatorname{cotk}_{m}^{(j)} b_{j}}{k_{m}^{(j)}}+\frac{1}{M_{j}}\right\}^{-1}
\end{aligned}
$$




$$
\begin{aligned}
& f_{j}\left(\bar{x}_{t}\right)=\sum_{m_{0}} \frac{M_{j} \cos K_{m}^{(j)}\left(b_{j}-y\right)}{K_{m}^{(j)} \operatorname{sinK} m_{b}^{(j)}} \sin \frac{m \pi}{2} \cos M_{j} \times J_{0}\left(M_{j} g\right) \\
& K_{m}^{(j)^{2}}=\zeta^{2}-M_{j}^{2} \\
& \Delta_{n}=\left\{\begin{array}{cc}
1 / 2 & n=0 \\
1 & n=1
\end{array}\right.
\end{aligned}
$$

with $m_{0}$ denoting the summation over odd $m$ only $(m=1,3,5 \ldots)$, and $J_{0}$ is the zeroth order Bessel function. Again the delta function should be understood in the sense of a generalized function.

\section{THE INPUT IMPEDANCE $Z$}

4.1. The Green's function terms $\tilde{K}_{1}^{(j)}$ and $\tilde{K}_{2}^{(j)}$ will lead to two distinct impedance contributions $z_{1}$ and $z_{2}$ which represent the ordinary and perturbation contributions to the probe input impedance. The total impedance is the sum of these two terms. Combining the form of the Green's function given by (4), with the impedance integral (1), yields $z=z_{1}+z_{2}$, where

$$
\begin{aligned}
& Z_{1}=\frac{-1}{2 \pi I_{t}^{2}} \int_{S} \int_{S^{\prime}} J(\bar{x}) \int_{-\infty}^{\infty} \tilde{K}_{1}^{(1)}\left(\bar{x}_{t}, \bar{x}_{t}^{\prime}\right) e^{i \alpha z} J\left(\bar{x}^{\prime}\right) d \alpha d s^{\prime} d s \\
& Z_{2}=\frac{-1}{2 \pi I_{t}^{2}} \iint_{S} J(\bar{x}) \int_{-\infty}^{\infty} \tilde{K}_{2}^{(1)}\left(\bar{x}_{t}, \bar{x}_{t}^{\prime}\right) e^{i \alpha z} J\left(\bar{x}^{\prime}\right) d \alpha d s^{\prime} d s
\end{aligned}
$$

The integrals defined for $Z_{1}$ and $Z_{2}$ are evaluated in Appendices $B$ and $C$ for $a$ centrally located probe. The approximate form found for $z_{1}$ depends upon two assumptions: (1) the probe is taken to be thin, i.e., $\pi t / 2 a_{1}<1$, and (2) the frequency is restricted so that $\left(k a_{1}\right)^{2}$ is small. More precisely, terms in $t$ of the order $\left(\pi t / 2 a_{1}\right)^{2}$ are discarded and terms in $k a_{1}$ of the order $\left(k / 2 M_{1}\right)^{4}$ 
are discarded. The evaluation of $z_{2}$ involves no approximations. However, $z_{2}$ remains in an integral form. The results of Appendices $B$ and $C$ are as follows

$$
\begin{array}{r}
z_{1} \approx \frac{i \omega \mu}{2 \pi b_{1} k^{2}} \tan \frac{2 k d}{2}\left\{\ln \left(\frac{4 a_{1}}{\pi t}\right)+\frac{a_{1}^{2} k^{2}}{\pi^{2}}(4.207175)\right. \\
\left.-2 k^{2} \sum_{n=1}^{\infty}\left(1-\frac{\sin ^{2} N_{1} d / 2}{\sin ^{2} k d / 2}\right)^{2} \frac{k_{0}\left[t\left(N_{1}^{2}-k^{2}\right)^{1 / 2}\right]}{N_{1}^{2}-k^{2}}\right\} \\
z_{2}=\frac{-i \omega \mu k^{2}}{4 a_{1}^{2}} \csc ^{2} k d \int_{-\infty}^{\infty} \frac{L(\alpha)}{\zeta^{2}} \sum m_{0} h_{m^{\prime}}(\alpha, d) \sum_{m_{m}} h_{m}(\alpha, d) J_{0}\left[t\left(\alpha^{2}+\mu_{1}^{2}\right)^{1 / 2}\right] d \alpha \\
n_{m}(\alpha, d)=\frac{M_{1} \sin \frac{m \pi}{2} J_{0}\left(M_{1} g\right)}{k_{m}^{(1)} \sin k_{m}^{(1)} b_{1}} \frac{\left(\cos k d-\cos k_{m}^{(1)} d\right)}{M_{1}^{2}+\alpha^{2}}
\end{array}
$$

where $K_{0}(x)$ is a modified Bessel function.

The perturbation term $z_{2}$ contains the radiation resistance due to the propagating TEM mode, which may be extracted by evaluating the half residues at $\alpha= \pm k$ due to the forward and backward propagating TEM modes. The TEM mode radiation resistance is found to be:

$$
R=\frac{\pi n_{0} k^{2}}{4 a_{1}^{2}} \csc ^{2} k d \quad L(k) \sum_{m_{0}} h_{m^{\prime}}(k, d) \sum_{m_{0}} h_{m}(k, d) J_{0}\left[t\left(k^{2}+M^{2}\right)^{1 / 2}\right]
$$

where $n_{0}=120 \pi$.

If we now let $a_{1}=a_{2}=a$ so that we are considering a normal TEM cell geometry, and recall that the characteristic impedance $Z_{C}$ of an RCTL is given by [4]

$$
z_{c}=\frac{n_{0} \pi}{8} L(k)
$$


The radiation resistance may be written

$$
R=\frac{2 k^{2}}{a^{2}} z_{c} \csc ^{2} k d \sum_{m_{0}} h_{m^{\prime}}(k, d) \sum_{m_{0}} h_{m}(k, d) J_{0}\left[t\left(k^{2}+M^{2}\right)^{1 / 2}\right]
$$

Because only the TEM mode is allowed to propagate, $R$ represents the total resistance. We may also express $Z$ as the sum of the radiation resistance $R$ and a reactance $i(X+\delta X)$, with $X$ and $\delta X$ defined by

$$
\begin{aligned}
& x=\frac{n_{0}}{2 \pi b_{1} k} \tan ^{2} \frac{k d}{2}\left\{\ln \left(\frac{4 a}{\pi t}\right)+\frac{a^{2} k^{2}}{\pi^{2}}(4.207175)\right. \\
& \left.-2 k^{2} \sum_{n=1}^{\infty}\left(1-\frac{\sin ^{2} N_{1} d / 2}{\sin ^{2} k d / 2}\right)^{2} \frac{k_{0}\left[t\left(N_{1}^{2}-k^{2}\right)^{1 / 2}\right]}{N_{1}^{2}-k^{2}}\right\} \\
& \delta X=\frac{-\omega \mu k^{2}}{4 a^{2}} \csc ^{2} k d P \int_{-\infty}^{\infty} \frac{L(\alpha)}{\zeta^{2}} \sum_{m} h_{m^{\prime}}(\alpha, d) \sum_{m} h_{m}(\alpha, d) J_{0}\left[t\left(\alpha^{2}+11^{2}\right)^{1 / 2}\right] d \alpha
\end{aligned}
$$

where the integral is now understood in the principal value sense. The dominant reactive contribution comes from the sum over $n$ and therefore the ractance is highly capacitive. The capacitive behavior is expected from comparisons to either a monopole in free space, or a similar probe in a rectangular guide. For typical TEM cell geometries, the principal value integral does not significantly add to the reactance. Therefore, a very good algebraic approximation for the reactance is simply $X$ which allows us to write $Z$ as follows:

$$
z \simeq R+i x
$$

where $R$ and $X$ are given respectively in (19) and (20). 


\section{NUMERICAL RESULTS AND DISCUSSIONS}

In order to check our result we would like to compare our expression to certain special cases for which solutions are known. The impedance $Z$ consists of three terms: ( $i$ ) the radiation resistance $R$ due to the propagating TEM mode, $(i i)$ the reactive ordinary rectangular waveguide contribution $z_{1}$, and (iii) the gap field perturbation reactance principal value integral. Ideally, since each of these terms has a distinct physical interpretation, each term should be checked individually. Because the probe is electrically short, the probe radiation resistance may be compared to that of a Hertzian dipole via a result given earlier by Tippet [1]. His expression for the radiation resistance of a Hertzian dipole involves the square of $I_{0} d l$ where $I$ is the current magnitude, $E_{0}$ is the TEM mode electric field distribution, and de is the effective dipole length. In the monopole case, the dipole quantity ( $I E_{0} d \ell$ ), may be replaced by an effective value which results from integrating the product $I(y) E_{0}(y)$ over the monople length. The two results agree up to the order of our approximations. Next, by letting the gap width go to zero $(g+0)$ thereby reducing the upper chamber to an ordinary rectangular waveguide, $z_{1}$ may be compared to a result given by collin [3]. If we allow the frequency to be large enough that the ordinary rectangular waveguide $\mathrm{TE}_{10}$ mode will propagate, then our result reduces exactly to that found by collin. Both these checks are detailed in Appendix $D$ for the special case when $a_{1}=a_{2}=a$.

The perturbation reactance term is more difficult to verify and is not comparable to any simple special case result. However, a good check of the self consistency of our expression exists. If we had allowed $k$ to be sufficiently large, such that $k^{2}>M_{1}^{2}$, the expression (12) for $Z_{1}$ would contain poles at $\alpha= \pm \beta_{10}(1)$, where $\beta_{10}^{(1)}=\left(k^{2}-M_{1}^{2}\right)^{1 / 2}$. This would imply an apparent mode propagating the same as an upper chamber $T E_{10}$ mode. Equation (15) for $Z_{2}$ would also imply the existence of this mode. However, the guide as a whole supports no such mode, therefore, we expect that the contributions from $z_{1}$ and $Z_{2}$, to this evident $T E\left(\frac{1}{0}\right)$ mode, should cancel out to the order of our previous 
approximations. It is found in Appendix $D$ that the radiation resistance due to a propagating TE $\left(\frac{1}{0}\right)$ mode in a rectangular waveguide of the dimensions of the upper chamber, is given by

$$
R=\frac{n_{0}}{2 a_{1} b_{1} k \beta_{10}^{(1)}} \tan ^{2} \frac{k d}{2}
$$

Now consider the perturbation contribution given by $z_{2}$ in $(15)$. In order to avoid the degeneracy due to an additional TE $\left\{\frac{1}{0}\right\}$ mode also propagating in the lower chamber, we refrain from letting the chamber widths be equal until after we have evaluated the pole contribution at $\alpha= \pm \beta_{10}^{(1)}$. As $\alpha$ approaches $\pm \beta_{10}^{(1)}$, we see that $k_{1}^{(1)}=\left(\beta_{10}^{(1) 2}-\alpha^{2}\right)^{1 / 2} \rightarrow 0$. Evaluating the half residues at $\alpha= \pm \beta_{10}^{(1)}$, we find

$$
\begin{gathered}
L(\alpha) \rightarrow \frac{2 a_{1} b_{1}\left(\beta_{10}^{(1) 2}-\alpha^{2}\right)}{\pi} \\
\zeta^{2} \rightarrow\left(\pi / 2 a_{1}\right)^{2} \\
h_{m}(\alpha, d) \rightarrow \frac{\pi(1-\cos k d)}{2 a_{1} b_{1} k^{2}\left(\beta_{10}^{(1) 2}-\alpha^{2}\right)} J_{0}\left(\frac{\pi g}{2 a_{1}}\right) \\
J_{0}\left[t\left(M_{1}^{2}+\alpha^{2}\right)^{1 / 2}\right] \rightarrow J_{0}(k t)
\end{gathered}
$$

Because both $\pi g / 2 a_{1}$ and $k t$ are small, the Bessel functions may be replaced by unity. Collecting results gives

$$
\begin{aligned}
\left.z_{2}\right|_{\alpha= \pm \beta}(1) & =\frac{-i \omega \mu k^{2}}{4 a_{10}^{2}} \csc ^{2} k d(-2 \pi i)_{\alpha \rightarrow \pm \beta} \lim _{10}(1) \frac{L(\alpha)}{\left(\pi / 2 a_{1}\right)^{2}} h_{1}^{2}\left(\beta_{10}^{(1)}, d\right) \\
& =\frac{-n_{0}}{2 a_{1} b_{1} k \beta_{10}^{(1)}} \tan ^{2} \frac{k d}{2}
\end{aligned}
$$


which indeed will cancel the unperturbed result given by expression (23). Thus the poles at $\alpha= \pm \beta_{10}^{(1)}$, that apparently imply a physically meaningless mode, do not contribute up to the order of our approximations. The impedance expressions will next be used to generate numerical data for typical TEM cell geometries.

The radiation resistance $R$ given by (19) is plotted in figure 3 for $g / a=0.1$ and 0.2 . The dimensions of the RCTL cross section are $a_{1}=a_{2}=1.0 \mathrm{~m}$, and $b_{1}=b_{2}=1.0 \mathrm{~m}$. The probe varies in length from $d / a=0.0$ to $d / a=0.95$, and is of radius $t=1 \mathrm{~mm}$. The frequency is set at $1 \mathrm{MHz}$. The curves show a basically parabolic dependence on $d$ with the main difference between the two curves resulting from the logarithmic dependence of $z_{c}$ on the gap size. This $d^{2}$ variation is significantly larger than the free-space probe radiation resistance which is proportional to $(k d)^{2}$, a frequency dependent quantity. This difference between the radiated power by a short monopole in free space and that delivered to a TEM mode has also been encountered in other types of problems such as the coupling between a dipole antenna and an infinite cable [8]. Figure 4 gives the dependence on $d$ of the ordinary rectangular waveguide reactance $X$. The same set of parameters is used to plot $x$, given by (20), as that used to plot $R$. The curve shows a highly capacitive contribution which is independent of the gap size. Again, this result may be compared to the free-space result for an electrically short monopole which is known as proportional to $-i(k d)^{-1}$ [9]. From (20), the dominant reactance behavior for small $k d$ is more like $-i(k d)^{-2}$. Thus, we find that the reactance of a monopole in a TEM cell is more highly capacitive than that in free space. Finally, the perturbation reactance $\delta X$ is plotted in figure 5 using (21), again for the same parameter set. We find that $|\delta X|<1 \Omega$ for the entire range of probe lengths. Thus $\delta x$ is at least three orders of magnitude less than $X$. Therefore, neglecting $\delta X$ will yield excellent results. The FORTRAN program used to compute $Z$ is listed in Appendix $E$. 


\section{CONCLUDING REMARKS}

This report has detailed the manner in which a coaxial probe excites a TEM ce11. The analysis utilizes three simplifying assumptions, which are (i) the gap is small, that is $G^{2} \ell n G<<1$ where $G=\pi g / 2 a$, ( $i i$ ) the probe is thin, or $\pi t / 2 a<<1$ where $t$ is the probe radius, and ( $i i i$ ) the frequency is not too high so that only the TEM mode propagates and $(k a)^{2}$ is small when compared to unity. None of the requirements is restrictive in terms of common TEM cell usage. The resulting expression for the probe input impedance consists of three distinct terms $R, X$, and $\delta X$, these being the radiation resistance due to the propagating TEM mode, the ordinary rectangular waveguide reactance, and the reactance perturbation due to the gap fields. The first two terms, $R$ and $X$, are computationally simple, with $R$ showing a basically $d^{2}$ dependence on probe length and $X$ giving a highly capacitive reactance. Although $\delta X$ consists of a nontrivial principal value integral, numerical results show that $|\delta x|<x$, and therefore $\delta x$ may be neglected. Thus a reasonably straightforward form for $Z$ is achieved which may be used to analyze probes inserted into a TEM cell either to measure, or excite fields as well as other types of feed lines.

\section{ACKNOWLEDGMENTS}

The authors are grateful to Dr. M. Kanda for his technical discussions during the course of this work. They also wish to thank C. K. S. Miller and $F$. X. Ries for their continuous interest and support. 


\section{REFERENCES}

[1] Tippet, J. C. Modal characteristics of rectangular coaxial strip line, Ph.D. Thesis, University of Colorado, Boulder, Colorado, 1978.

[2] Sreenivasiah, I.; Chang, D. C.; Ma, M. T. Characterization of electrically small radiating sources by tests inside a transmission line cell. Nat. Bur. Stand. (U.S.) Tech. Note 1017; 1980.

[3] Collin, R. E. Field theory of guided waves. New York: McGraw-Hi11; 1960.

[4] Wilson, P. F.; Chang, D. C.; Ma, M. T. Excitation of a TEM cell by a vertical electric hertzian dipole. Nat. Bur. Stand. (U.S.) Tech. Note $1037 ; 1981$.

[5] Tricomi, F. G. Integral equations, chapter IV. New York: Interscience Publishers, Inc.; 1957.

[6] Abramowitz, M.; Stegun, I. A., ed. Handbook of mathematical functions with formulas, graphs, and mathematical tables. Nat. Bur. Stand. (U.S.) Appl. Math. Ser. 55; 1964.

[7] Gradshteyn, I. S.; Ryzhik, I. M. Tables of integrals, series, and products. New York: Academic Press; 1965.

[8] Hill, D.A.; Wait, J.R. Coupling Between a dipole antenna and an infinite cable over an ideal ground plane. Radio Science, 12, No. 2: 231-238; 1977 March-April.

[9] Tai, C.T. Characteristics of linear antenna elements, Chapter 3 of Antenna Engineering Handbook, H. Jasik, Ed. New York, NY: McGraw-Hi11; 1961. 


\section{APPENDIX A}

$$
\text { DERIVATION OF } \tilde{\mathrm{K}}_{1}^{(j)} \text { and } \tilde{\mathrm{K}}_{2}^{(j)}
$$

Generalizing the chamber widths involves some modifications in the expressions derived in NBS Technical Note 103 [4]. For the most part, this involves nothing more than replacing a by $a_{j}$. The details will be briefly outlined beginning with the TE type mode kernel which becomes

$$
\begin{aligned}
& Q^{(h)}\left(t, t^{\prime}\right)=Q_{s}^{(h)}\left(t, t^{\prime}\right)-Q_{f}^{(h)}\left(t, t^{\prime}\right) \\
& Q_{S}^{(h)}\left(t, t^{\prime}\right) \approx-\frac{1}{\pi} \ln \left[\left(\frac{\pi}{4 a_{1}}\right)\left(\frac{\pi}{4 a_{2}}\right)\left|t^{2}-t^{\prime 2}\right|\right] \\
& Q_{f}^{(h)}\left(t, t^{\prime}\right) \approx \sum_{j=1}^{2} \frac{1}{a_{j}} \sum_{m o}\left[\frac{\cot k_{m}^{(j)} b_{j}}{k_{m}^{(j)}}+\frac{1}{M_{j}}\right] \equiv Q_{f}
\end{aligned}
$$

where $t=a-x, \quad t^{\prime}=a-x^{\prime}, \quad a=\min \left(a_{1}, a_{2}\right), \quad K_{m}^{(j) 2}=\zeta^{2}-M_{j}^{2}, M_{j}=m \pi / 2 a_{j}$, and mo refers to the summation over odd $m$. This kernel will result in the following solution for the unknown gap field

$$
\begin{aligned}
& f^{(h)}\left(t^{\prime}\right)=\frac{-I d \ell}{2 a_{1} \sqrt{g^{2}-t^{\prime 2}}} L(\alpha) f_{l}\left(\bar{x}_{t}^{\prime}\right) \\
& L(\alpha)=\left[\ln \left(\frac{8 \sqrt{a_{1} a_{2}}}{\pi g}\right)+\frac{\pi}{2} Q_{f}\right]^{-1} \\
& f_{j}\left(\bar{x}_{t}\right)=\sum_{m o} \frac{M_{j} \cos K_{m}^{(j)}\left(b_{j}-y\right)}{K_{m}^{(j)} \sin k_{m}^{(j)} b_{j}} \sin \frac{m \pi}{2} \cos M_{j} x_{0} J_{0}\left(M_{j} g\right)
\end{aligned}
$$

where we recall that $\bar{x}_{t}^{\prime}$ is the dipole location. The above solution assumes that the dipole is centrally located.

The $y$-component of the electric field in the upper, and lower chambers may now be found. Both TE and TM type unperturbed (ordinary) modes, as we11 as TE type perturbed modes will contribute significantly to $\tilde{E}_{y}^{(j)}$. The unperturbed modes exist only in the upper chamber. Denoting the unperturbed 
contribution by $\tilde{K}_{1}^{(j)}$, and the gap perturbation contribution by $\tilde{K}_{2}^{(j)}$, and referring to Appendix C of NBS Technical Note 1037 [4], we find

$$
\tilde{K}_{i}^{(j)}\left(\bar{x}_{t}, \bar{x}_{t}^{\prime}\right)=\delta_{i j}\left\{\frac{-i \omega \mu}{\zeta^{2}} \partial_{x} \tilde{H}_{z}^{(j)}\left(\bar{x}_{t}, \bar{x}_{t}^{\prime}\right)+\frac{i \alpha}{\zeta^{2}} \partial_{y} \tilde{E}_{z}^{(j)}\left(\bar{x}_{t}, \bar{x}_{t}^{\prime}\right)\right\}
$$

Suitabiy modifying equation (49) of NBS Technical Note 1037 [4] yields

$$
\tilde{K}_{2}^{(j)}\left(\bar{x}_{t}, \bar{x}_{t}^{t}\right)=\frac{i \omega \mu \pi}{2 a_{1} a_{j} \zeta^{2}} \text { Id } L(\alpha) f_{j}\left(\bar{x}_{t}\right) f_{1}\left(\bar{x}_{t}^{\prime}\right)
$$

Taking the proper derivatives required in (a-7) and substituting the proper forms for $\tilde{E}_{z}^{(j)}$, and $\tilde{H}_{Z}^{(j)}$ allows $\tilde{K}_{1}^{(j)}$ to be written as follows.

$$
\begin{aligned}
& \tilde{K}_{i}^{(j)}\left(\bar{x}_{t}, \bar{x}_{t}^{\prime}\right)=\delta_{1 j} \frac{i \omega \mu}{\zeta^{2}} \text { Id } \sum_{m, n=0}^{\infty} \frac{\Delta_{n} M_{j}^{2}+\frac{\alpha^{2}}{k^{2}} N_{j}^{2}}{M_{j}^{2}+N_{j}^{2}-\zeta^{2}} g_{m n}^{(j)}\left(\bar{x}_{t}, \bar{x}_{t}^{\prime}\right) \\
& g_{m n}^{(j)}\left(\bar{x}_{t}, \bar{x}_{t}^{\prime}\right)=\left(\frac{2}{a_{j} b_{j}}\right) \sin M_{j}\left(x+a_{j}\right) \sin \left(x^{\prime}+a_{j}\right) \cos N_{j} y \cos N_{j} y^{\prime}
\end{aligned}
$$

where

$$
\Delta_{n}= \begin{cases}\frac{1}{2} & n=0 \\ 1 & n \geq 1\end{cases}
$$

and $N_{j}=n \pi / b_{\sim}$. It will prove convenient to extract the delta function nature in $\tilde{K}_{j}^{(j)}$ which occurs when $\bar{x}_{t} \rightarrow \bar{x}_{t}^{1}$. Notice that

$$
\Delta_{n} M_{j}^{2}+\frac{\alpha^{2}}{k^{2}} N_{j}^{2}=\Delta_{n} \frac{\alpha^{2}}{k^{2}}\left[M_{j}^{2}+N_{j}^{2}-\zeta^{2}+\frac{\zeta^{2}}{\alpha^{2}} M_{j}^{2}+\zeta^{2}\right]
$$

Therefore $\tilde{K}_{j}^{(j)}$ becomes

$$
\begin{aligned}
& \tilde{K}_{1}^{(j)}\left(\bar{x}_{t}, \bar{x}_{t}^{\prime}\right)=\delta_{1 j} \frac{i \omega \mu}{k^{2}} \operatorname{Id} \ell\left\{\frac{\alpha^{2}}{\zeta^{2}} \sum_{m, n=0}^{\infty} \Delta_{n} g_{m n}^{(j)}\left(\bar{x}_{t}, \bar{x}_{t}^{\prime}\right)\right. \\
& \left.+\sum_{m, n=0}^{\infty} \frac{\Delta_{n}\left[M_{j}^{2}+\alpha^{2}\right]}{M_{j}^{2}+N_{j}^{2}-\zeta^{2}} g_{m n}^{(j)}\left(\bar{x}_{t}, \bar{x}_{t}^{1}\right)\right\}
\end{aligned}
$$

If we expand a delta function in a double Fourier series over a chamber cross section we will find

$$
\delta\left(\bar{x}_{t}-\bar{x}_{t}^{\prime}\right)=\sum_{m, n=0}^{\infty} \Delta_{n} g_{m n}^{(j)}\left(\bar{x}_{t}, \bar{x}_{t}^{\prime}\right)
$$


Therefore $\tilde{K}_{1}^{(j)}$ becomes

$$
\begin{gathered}
\tilde{k}_{1}^{(j)}\left(\bar{x}_{t}, \bar{x}_{t}^{\prime}\right)=\delta \gamma_{1 j} \frac{i \omega_{\mu}}{k^{2}} \operatorname{Id} \ell\left\{\frac{\alpha^{2}}{\zeta^{2}} \delta\left(\bar{x}_{t}-\bar{x}_{t}^{1}\right)\right. \\
\left.+\sum_{m, n=0}^{\infty} \frac{\Delta_{n}\left[M_{j}^{2}+\alpha^{2}\right]}{M_{j}^{2}+N_{j}^{2}-\zeta^{2}} g_{m n}^{(j)}\left(\bar{x}_{t}, \bar{x}_{t}^{1}\right)\right\}
\end{gathered}
$$

$\tilde{K}_{1}^{(j)}$ and $\tilde{K}_{2}^{(j)}$ represent the $y$-component of the electric field excited by a symmetrically located VED at $\bar{x}_{t}^{\prime}$. There remain only two small changes to adapt this result to the probe excitation problem. First, the probe carries a negative $y$-directed current. Thus Idl $\rightarrow-I d l$. Second, the Green's function represents results from a unit strength dipole. Therefore $-I d \ell \rightarrow-1$. Therefore $\tilde{K}_{1}^{(j)}$ and $\tilde{K}_{2}^{(j)}$ finally become

$$
\begin{aligned}
& \tilde{k}_{1}^{(j)}\left(\bar{x}_{t}, \bar{x}_{t}\right)=-\delta j \frac{i \omega \mu}{k^{2}}\left\{\frac{\alpha^{2}}{\zeta^{2}} \delta\left(\bar{x}_{t}-\bar{x}_{t}^{\prime}\right)\right. \\
& \left.+\sum_{m, n=0}^{\infty} \frac{\Delta_{n}\left[M_{j}^{2}+\alpha^{2}\right]}{M_{j}^{2}+N_{j}^{2}-\zeta^{2}} g_{m n}^{(j)}\left(\bar{x}_{t}, \bar{x}_{t}^{\prime}\right)\right\} \\
& \tilde{k}_{2}^{(j)}\left(\bar{x}_{t}, \bar{x}_{t}^{\prime}\right)=\frac{i \omega \mu \pi}{2 a_{1} a_{j} \zeta^{2}} L(\alpha) f_{j}\left(\bar{x}_{t}\right) f_{l}\left(\bar{x}_{t}^{\prime}\right)
\end{aligned}
$$


The integral defined for $z_{1}$ is to be evaluated.

$$
\begin{aligned}
& z_{1}=\frac{-1}{2 \pi I_{t}^{2}} \int_{S^{\prime}} \int_{S^{\prime}} J(\bar{x}) \int_{-\infty}^{\infty} \tilde{k}_{1}^{(1)}\left(\bar{x}_{t}, \bar{x}_{t}^{\prime}\right) e^{i \alpha z} J\left(\bar{x}^{\prime}\right) d \alpha d s^{\prime} d s \\
& \tilde{K}_{1}^{(1)}\left(\bar{x}_{t}, \bar{x}_{t}^{\prime}\right)=-\frac{i \omega \mu}{k^{2}}\left\{\frac{\alpha^{2}}{\zeta^{2}} \delta\left(\bar{x}_{t}-\bar{x}_{t}^{\prime}\right)\right. \\
&\left.\quad+\sum_{m, n=0}^{\infty} \Delta_{n} \frac{M_{1}^{2}+\alpha^{2}}{M_{1}^{2}+N_{1}^{2}-\zeta^{2}} g_{m n}^{(1)}\left(\bar{x}_{t}, \bar{x}_{t}^{\prime}\right)\right\}
\end{aligned}
$$

The $\alpha$-integration may be performed first, which requires evaluating the following pair of integrals.

$$
\begin{aligned}
& I_{1}=\int_{-\infty}^{\infty} \frac{\alpha^{2}}{\zeta^{2}} \delta\left(\bar{x}_{t}-\bar{x}_{t}^{\prime}\right) e^{i \alpha z} d \alpha \\
& I_{2}=\int_{-\infty}^{\infty} \sum_{m, n=0}^{\infty} \Delta_{n} \frac{M_{1}^{2}+\alpha^{2}}{M_{1}^{2}+N_{1}^{2}-\zeta^{2}} g_{m n}^{(1)}\left(\bar{x}_{t}, \bar{x}_{t}^{\prime}\right) e^{i \alpha z} d \alpha
\end{aligned}
$$

In each integral we will deform into the upper $\alpha$ half plane for $z>0$ (forward waves), and into the lower $\alpha$ half plane for $z<0$ (backward waves). For $I_{1}$, we find

$$
I_{1}=-i \pi k \delta\left(\bar{x}_{t}-\bar{x}_{t}^{\prime}\right) e^{i k|z|}
$$

The denominator of $\mathrm{I}_{2}$ may be factored as follows.

$$
\begin{aligned}
& M_{1}^{2}+N_{1}^{2}-\zeta^{2}=\alpha^{2}+\Gamma_{m n}^{(1)^{2}} \\
& \Gamma_{m n}^{(1)}=\left[M_{1}^{2}+N_{1}^{2}-k^{2}\right]^{\frac{1}{2}}
\end{aligned}
$$


Thus we see that a simple pole arises in the $I_{2}$ integrand when $\alpha= \pm{ }_{i} \Gamma_{m n}(1)$. Again deforming as for $I_{1}$ gives the following result.

$$
I_{2}=\pi \sum_{m, n=0}^{\infty} \Delta_{n} \frac{k^{2}-N_{1}^{2}}{\Gamma_{m n}^{(1)}} g_{m n}^{(1)}\left(\bar{x}_{t}, \bar{x}_{t}^{\prime}\right) e^{-\Gamma_{m n}^{(1)}|z|}
$$

We next proceed to the integration over the current distributions $J(\bar{x})$ and $J\left(\bar{x}^{\prime}\right)$. The source current $J\left(\bar{x}^{\prime}\right)$ will be concentrated on an idealized linear antenna located at the origin, i.e. $\bar{x}_{t}^{\prime}=\left(0, y^{\prime}\right)$. Therefore $\bar{x}_{t} \neq \bar{x}_{t}$ on $S$ and the delta function term will not contribute to $z_{7}$. Thus $z_{1}$ reduces to

$$
Z_{1}=\frac{i \omega \mu}{2 k^{2} I_{t}^{2}} \sum_{m, n=0}^{\infty} \Delta_{n} \frac{k^{2}-N_{l}^{2}}{\Gamma_{m n}^{(1)}} \int_{S S^{\prime}} J(\bar{x}) g_{m n}^{(1)}\left(\bar{x}_{t}, \bar{x}_{t}^{\prime}\right) J\left(\bar{x}^{\prime}\right) e^{-\Gamma_{m n}^{(1)}|z|} d s^{\prime} d s
$$

The simpler integration over the source current will be considered first. We recal1 that the assumed current density on the probe will be

$$
J(\bar{x})=\frac{I_{0}}{2 \pi t} \sin k\left(b_{1}-d-y\right) \delta(\rho-t)
$$

For the $\delta^{\prime}$ integration we let $t^{\prime} \rightarrow 0$, and therefore

$$
\begin{aligned}
& \int_{S^{\prime}} J\left(\bar{x}^{\prime}\right) g_{m n}^{(1)}\left(\bar{x}_{t}, \bar{x}_{t}^{\prime}\right) d s^{\prime} \\
& \quad=I_{3}\left(\frac{2}{a_{7} b_{7}}\right) I_{0} \sin M_{7}\left(x+a_{7}\right) \sin \frac{m \pi}{2} \cos N_{7} y e^{-\Gamma_{m n}^{(1)}|z|}
\end{aligned}
$$

where the integral $I_{3}$ is given by

$$
I_{3}=\int_{b_{1}-d}^{b_{7}} \sin k\left(b_{1}-d-y^{\prime}\right) \cos N_{1} d^{\prime} d y^{\prime}=\frac{k \cos n \pi\left(\cos k d-\cos N_{7} d\right)}{k^{2}-N_{1}^{2}}(b-12)
$$

Because of the factor $\sin m \pi / 2$, only the summation over odd $m$ will contribute.

Therefore $z_{1}$ takes the form

$$
Z_{1}=\frac{i \omega \mu I_{0}}{a_{1} b_{1} k I_{t}^{2}} \sum_{m 0, n=0}^{\infty} \frac{\Delta_{n} \cos n \pi}{\Gamma_{m n}^{(1)}}\left(\cos k d-\cos N_{1} d\right) \int_{S} J(\bar{x}) \cos M_{1} x \cos N_{1} y
$$


The integration over $S$ may be split into separate integrations over $y$ and $\theta$. The $y$-integration has already been encountered and we find

$$
\int_{S} J(\bar{x}) \cos M_{1} x \cos N_{1} y e^{-\Gamma_{m n}^{(1)}|z|} d s=\frac{I_{0} I_{3}}{2 \pi t} \int_{0}^{2 \pi} \cos \left(M_{1} t \cos \theta\right) e^{-\Gamma_{m n}^{(1)} t|\sin \theta|} t d \theta
$$

If we now interchange the remaining theta integration with the double summation, $z_{1}$ may be written

$$
\begin{aligned}
Z_{1}= & \frac{i \omega \mu I_{0}^{2}}{\pi a_{1} b_{1} I_{t}^{2}} \int_{0}^{\pi}\left\{\sum_{n=0}^{\infty} \Delta_{n} \frac{\left(\cos k d-\cos N_{1} d\right)^{2}}{k^{2}-N_{1}^{2}}\right. \\
& \left.\times \sum_{m 0} \frac{1}{\Gamma_{m n}^{(1)}} \cos \left(M_{1} t \cos \theta\right) e^{-\Gamma_{m n}^{(1)} t \sin \theta}\right\} d \theta
\end{aligned}
$$

where we have noted that the theta integration is symmetric about $\pi$. Let us now consider the sum over $\mathrm{m}$

$$
S_{n}(\theta)=\sum_{m 0} \frac{1}{\Gamma_{m n}^{(1)}} \cos \left(M_{1} t \cos \theta\right) e^{-\Gamma_{m n}^{(1)} t \sin \theta}
$$

The dominant series will be for $n=0 . S_{0}(\theta)$ may be expressed as follows.

$$
S_{0}(\theta)=\left.\operatorname{Re} \sum_{m 0} \frac{e^{i M_{1} t \cos \theta-\Gamma_{m n}^{(1)} t \sin \theta}}{\Gamma_{m n}^{(1)}}\right|_{n=0}
$$

For large $m$ we see that $\Gamma_{m 0}^{(1)} \sim M_{1}$. Adding and subtracting the asymptotic form of the summand will thus give

$$
\begin{aligned}
S_{0}(\theta) & =\operatorname{Re} \sum_{m 0} \frac{e^{M_{1} t(i \cos \theta-\sin \theta)}}{M_{1}} \\
& +\operatorname{Re} \sum_{m 0} e^{i M_{1} t \cos \theta}\left[\frac{e^{-\Gamma_{m n}^{(1)} t \sin \theta}}{\Gamma_{m n}^{(1)}}-\frac{e^{-M_{1} t \sin \theta}}{M_{1}}\right]_{n=0}
\end{aligned}
$$

where the first series represents the major contribution. The first series may be summed 


$$
\sum_{m_{0}} \frac{e^{i M_{1} t\left(e^{i \theta}\right)}}{M_{1}}=\frac{-a_{1}}{\pi} \ln \left[\tan \left(\frac{\pi t e^{i \theta}}{4 a_{1}}\right)\right]+\frac{i a_{1}}{2}
$$

We now assume that the probe is thin, $\pi t / 4 a_{1}<1$, which allows us to replace the tangent by its argument

$$
\ln \left[\tan \left(\frac{\pi t e^{i \theta}}{4 a_{1}}\right)\right] \approx \ln \left(\frac{\pi t}{4 a_{1}} e^{i \theta}\right)=\ln \left(\frac{\pi t}{4 a_{1}}\right)+i \theta
$$

If we now take the real part of the first sum in (b-18) we see that

$$
S_{0}(\theta)=\frac{a_{1}}{\pi} \ln \left(\frac{4 a_{1}}{\pi t}\right)+\operatorname{Re} \sum_{m_{0}} e^{i M_{1} t \cos \theta}\left[\frac{e^{-\Gamma_{m n}^{(1)} t \sin \theta}}{\Gamma_{m n}^{(1)}}-\frac{e^{-M_{1} t \sin }}{M_{1}}\right]_{n=0}
$$

The correction series will be considered next. Again consider $\mathrm{F}_{\mathrm{mo}}(1)$

$$
\Gamma_{\mathrm{mo}}^{(1)}=M_{1}\left(1-k^{2} / M_{1}^{2}\right)^{\frac{1}{2}} \approx M_{1}\left(1-k^{2} / 2 M_{1}^{2}\right)=M_{1}(1-\varepsilon)
$$

where $\varepsilon=k^{2} / 2 M_{1}^{2}$ is assumed to be smal1. The correction series may be written

$$
\sum_{m o} \frac{e^{M_{1} t(i \cos \theta-\sin \theta)}}{M_{1}}\left[\frac{e^{M_{1} t \varepsilon \sin \theta}}{1-\varepsilon}-1\right]
$$

For $\varepsilon$ sma 17 , we have $(1-\varepsilon)^{-1} \approx 1+\varepsilon$. This series converges very rapidly, thus only a few significant terms may be considered. Because $\pi t / 2 a_{1} \ll 1$ for these few terms, the exponents may be replaced by unity with little error giving

$$
\sum_{m_{0}} \frac{\varepsilon}{M_{1}}=\frac{k^{2}}{2} \sum_{m_{0}} \frac{1}{M_{1}^{3}}=\frac{4 a_{1}^{3} k^{2}}{\pi^{3}} \sum_{\text {mo }} \frac{1}{m^{3}}
$$

The sum of the inverse cube over odd $m$ may be done in terms of the Riemann Zeta function. Recalling that

$$
\zeta(s)=\sum_{k=1}^{\infty} k^{-s}
$$

For $\operatorname{Re}(s)>1$, we see that our expression may be written

$$
\sum_{m 0} \frac{1}{m^{3}}=\sum_{m=1}^{\infty} \frac{1}{m^{3}}-\sum_{m=1}^{\infty} \frac{1}{(2 m)^{3}}=\frac{7}{8} \zeta(3)
$$


The Riemann-Zeta function is well tabulated, and from [6] we find

$$
\zeta(3) \approx 1.20205
$$

Collecting these results we have the following for the dominant series $\mathrm{s}_{0}(\theta)$

$$
S_{0}(\theta) \approx \frac{a_{1}}{\pi} \ln \left(\frac{4 a_{1}}{\pi t}\right)+\frac{a_{1}^{3} k^{2}}{\pi^{3}}(4.207175)
$$

The remaining series $S_{n}(\theta)$ for $n \geq 1$ will be summed in a manner similar to that used by collin [3] in treating the probe excitation of a rectangular waveguide. We will make use of the following Poisson summation formula: if $f(x)$ is a square integrable function, then

$$
\sum_{n=-\infty}^{\infty} f(n \alpha)=\frac{1}{\alpha} \sum_{n=-\infty}^{\infty} F\left(\frac{2 \pi n}{\alpha}\right)
$$

where $F(\omega)$ is the Fourier-transform of $f(x)$ defined by

$$
F(\omega)=\int_{-\infty}^{\infty} f(x) e^{i \omega x} d x
$$

Our particular problem involves the function $f(x)$ defined by

$$
\begin{aligned}
& f(x)=e^{i x t \cos \theta-\Gamma_{m n}^{(1)}(x) t \sin \theta} / \Gamma_{m n}^{(1)}(x) \\
& \Gamma_{m n}^{(1)}(x)=\left[x^{2}+N_{1}^{2}-k^{2}\right]^{\frac{1}{2}}
\end{aligned}
$$

This function is clearly $L_{2}$ so Poisson's summation formula applies. The Fourier transform is given by

$$
F(\omega)=\int_{-\infty}^{\infty} \frac{e^{-\Gamma_{m n}^{(1)}(x) t \sin \theta}}{\Gamma_{m n}^{(1)}(x)} e^{i x(t \cos \theta+\omega)} d x
$$

If we now rewrite the complex exponential, and notice that $\Gamma_{m n}^{(1)}(x)$ is even in $x$, then $F(\omega)$ takes the form

$$
F(\omega)=2 \int_{0}^{\infty} \frac{e^{-\Gamma_{m}^{(1)}(x) t \sin \theta}}{\Gamma_{m n}^{(1)}(x)} \cos ((t \cos \theta+\omega) x) d x
$$


This integral may be evaluated in terms of the modified Bessel function $\mathrm{K}_{0}[7]$ with the result

$$
F(\omega)=2 K_{0}\left[\left(N_{1}^{2}-k^{2}\right)^{\frac{1}{2}}\left(t^{2} \sin ^{2} \theta+(t \cos \theta+\omega)^{2}\right)^{\frac{1}{2}}\right]
$$

If we now return to the definition of $S_{n}(\theta)$ we see that

$$
S_{n}(\theta)=\frac{1}{2} \sum_{m 0=-\infty}^{+\infty} f\left(\frac{m \pi}{2 a_{1}}\right)=\frac{1}{2} \sum_{m=-\infty}^{\infty}\left\{f\left(\frac{m \pi}{2 a_{1}}\right)-f\left(\frac{m \pi}{a_{1}}\right)\right\}
$$

Thus applying Poisson's summation formula we have

$$
S_{n}(\theta)=\frac{1}{2} \sum_{m=-\infty}^{\infty}\left\{\frac{2 a_{1}}{\pi} F\left(4 a_{1} m\right)-\frac{a_{1}}{\pi} F\left(2 a_{1} m\right)\right\}
$$

which in terms of modified Bessel functions $K_{0}$ becomes

$$
\begin{aligned}
S_{n}(\theta) & =\frac{a_{1}}{\pi} \sum_{m=-\infty}^{\infty}\left\{2 k_{0}\left[\left(N_{7}^{2}-k^{2}\right)^{\frac{1}{2}}\left(\left(4 a_{1} m\right)^{2}+8 a_{1} m t \cos \theta+t^{2}\right)^{\frac{1}{2}}\right]\right. \\
& \left.-k_{0}\left[\left(N_{7}^{2}-k^{2}\right)^{\frac{1}{2}}\left(\left(2 a_{7} m\right)^{2}+4 a_{1} m t \cos \theta+t^{2}\right)^{\frac{1}{2}}\right]\right\}
\end{aligned}
$$

The modified Bessel function decays very rapidly so the only significant contribution comes from $m=0$. Thus

$$
S_{n}(\theta) \approx \frac{a_{1}}{\pi} K_{0}\left[\left(N_{1}^{2}-k^{2}\right)^{\frac{1}{2}} t\right] \quad(n \geq 1)
$$

Returning to the integral for $z_{7}$ in $(b-15)$ we have the following

$$
z_{7} \approx \frac{i \omega \mu I_{0}^{2}}{\pi a_{1} b_{7} I_{t}^{2}} \int_{0}^{\pi}\left\{\frac{(1-\cos k d)^{2}}{2 k^{2}} S_{0}(\theta)+\sum_{n=1}^{\infty} \frac{\left(\cos k d-\cos N_{7} d\right)^{2}}{k^{2}-N_{1}^{2}} S_{n}(\theta)\right\} d \theta
$$

Note that the approximate form for $S_{n}(\theta)$ in $(b-39)$ is now independent of $\theta$. Thus combining all these results, we have

$$
\begin{array}{r}
Z_{1} \approx \frac{i \omega \mu I_{0}^{2}}{2 \pi b_{7} k^{2} I_{t}^{2}}(I-\cos k d)^{2}\left\{\ln \left(\frac{4 a_{1}}{\pi t}\right)+\frac{a_{7}^{2} k^{2}}{\pi^{2}}(4.207175)\right. \\
\left.-2 k^{2} \sum_{n=1}^{\infty}\left(1-\frac{\sin ^{2} N_{7} d / 2}{\sin ^{2} k d / 2}\right)^{2} \frac{k_{0}\left[t\left(N_{7}^{2}-k^{2}\right)^{1 / 2}\right]}{N_{7}^{2}-k^{2}}\right\}
\end{array}
$$


Note that $z_{1}$ given in $(b-41)$ is approximate, based on two assumptions that the probe is thin $\left(\pi t / 2 a_{1} \ll 1\right)$ and that the frequency is not too high $\left(k^{2} a_{1}^{2}<1\right)$.

Finally, we note that the total current at the aperture $\left(y=b_{7}\right)$ is given by $I_{t}=-I_{0} \operatorname{sink} d$. Thus,

$$
\begin{aligned}
& z_{1} \approx \frac{i \omega \mu}{2 \pi b_{1} k^{2}} \tan ^{2} \frac{k d}{2}\left\{\ln \left(\frac{4 a}{\pi t}\right)+\frac{a_{1}^{2} k^{2}}{\pi^{2}}(4.207175)\right. \\
& \left.\quad-2 k^{2} \sum_{n=1}^{\infty}\left(1-\frac{\sin ^{2} N_{1} d / 2}{\sin ^{2} k d / 2}\right)^{2} \quad \frac{k_{0}\left[t\left(N_{1}^{2}-k^{2}\right)^{\frac{1}{2}}\right]}{N_{1}^{2}-k^{2}}\right\} .
\end{aligned}
$$


The integral defined for $z_{2}$ in (13) is to be evaluated.

$$
z_{2}=\frac{-1}{2 \pi I_{t}^{2}} \int_{S} \int_{S^{\prime}} J(\bar{x}) \int_{-\infty}^{\infty} \tilde{K}_{2}^{(1)}\left(\bar{x}_{t}, \bar{x}_{t}^{\prime}\right) e^{i \alpha z} J\left(\bar{x}^{\prime}\right) d \alpha d s^{\prime} d s
$$

where

and

$$
\begin{aligned}
& \tilde{K}_{2}^{(1)}\left(\bar{x}_{t}, \bar{x}_{t}^{\prime}\right)=\frac{i \omega \mu \pi}{2 a_{1}^{2} \zeta^{2}} L(\alpha) f_{1}\left(\bar{x}_{t}\right) f_{1}\left(\bar{x}_{t}^{\prime}\right) \\
& L(\alpha)=\left\{\ln \left(\frac{8 \sqrt{a_{1} a_{2}}}{\pi g}\right)+\frac{\pi}{2} \sum_{j=1}^{2} \frac{1}{a_{j}} \sum_{m o}\left[\frac{\cot k_{m}^{(j)_{b}}}{k_{m}^{(j)}}+\frac{1}{M_{j}}\right]\right\}^{-1}
\end{aligned}
$$

$$
f_{1}\left(\bar{x}_{t}\right)=\sum_{m 0} \frac{M_{1} \cos k_{m}^{(1)}\left(b_{1}-y\right)}{k_{m}^{(1)} \sin k_{m}^{(1)} b_{1}} \sin \frac{m \pi}{2} \cos M_{1} \times J_{0}\left(M_{1} g\right)
$$

If we define two integrals $\mathrm{I}_{4}$ and $\mathrm{I}_{5}$ via

$$
\begin{aligned}
& I_{4}=\int_{S^{\prime}} J\left(\bar{x}^{\prime}\right) f_{7}\left(\bar{x}_{t}^{\prime}\right) d s^{\prime} \\
& I_{5}=\int_{S} J(\bar{x}) f_{7}\left(\bar{x}_{t}\right) e^{i \alpha z} d s
\end{aligned}
$$

and interchange the $\alpha$ - and surface integrations, we may write $z_{2}$ in the following form

$$
Z_{2}=\frac{-i \omega \mu}{4 a_{7}^{2} I_{t}^{2}} \int_{-\infty}^{\infty} \frac{L(\alpha)}{\zeta^{2}} I_{4} I_{5} d \alpha
$$

The integrals $\mathrm{I}_{4}$ and $\mathrm{I}_{5}$ now need to be evaluated. 
The assumed current distribution is given by (2). Therefore $\mathrm{I}_{4}$ becomes

$$
I_{4}=I_{0} \sum_{m 0} \frac{M_{1} \sin \frac{m \pi}{2} J_{0}\left(M_{1} g\right)}{k_{m}^{(1)} \sin k_{m}^{(1)} b_{1}} \int_{b_{1}-d}^{b_{7}} \sin k\left(b_{1}-d-y^{\prime}\right) \cos k_{m}^{(1)}\left(b_{1}-y^{\prime}\right) d y^{\prime}
$$

Performing the $y$-integration gives

$$
I_{4}=k_{0} \sum_{m 0} \frac{M_{1} \sin \frac{m \pi}{2} J_{0}\left(M_{1} g\right)}{k_{m}^{(1)} \sin k_{m}^{(1)} b_{1}} \cdot \frac{\left(\cos k d-\cos K_{m}^{(1)} d\right)}{M_{1}^{2}+\alpha^{2}}
$$

The integral $I_{5}$ is similar to $I_{4}$ except that an additional azimuthal integration around a probe must be performed. The $y$-integration is the same as in $\mathrm{I}_{4}$ so $\mathrm{I}_{5}$ becomes

$$
\begin{aligned}
I_{5} & =\frac{k_{0}}{2 \pi t} \sum_{m 0} \frac{M_{1} \sin \frac{m \pi}{2} J_{0}\left(M_{1} g\right)}{k_{m}^{(1)} \sin k_{m}^{(1)} b_{1}} \frac{\left(\cos k d-\cos k_{m}^{(1)} d\right)}{M_{1}^{2}+\alpha^{2}} \\
& \times \int_{0}^{2 \pi} \cos \left(M_{1} t \cos \theta\right) e^{i \alpha t \sin \theta} t d \theta
\end{aligned}
$$

If we designate the theta integration by $H_{m}(t)$, then letting $\theta \rightarrow 2 \pi-\theta$ in the interval $[\pi, 2 \pi]$ allows $H_{m}(t)$ to be written

$$
H_{m}(t)=2 t \int_{0}^{\pi} \cos \left(M_{1} t \cos \theta\right) \cos (\alpha t \sin \theta) d \theta
$$

The second cosine term may be expanded in terms of Bessel functions [7]

$$
\cos (\alpha t \sin \theta)=J_{0}(\alpha t)+2 \sum_{k=1}^{\infty} J_{2 k}(\alpha t) \cos 2 k \theta
$$

Interchanging the sum and integral gives

$$
\begin{aligned}
H_{m}(t) & =2 t\left\{J_{0}(\alpha t) \int_{0}^{\pi} \cos \left(M_{7} t \cos \theta\right) d \theta\right. \\
& \left.+2 \sum_{k=1}^{\infty} J_{2 k}(\alpha t) \int_{0}^{\pi} \cos 2 k \theta \cos \left(M_{7} t \cos \theta\right) d \theta\right\}
\end{aligned}
$$

Again consulting [7] allows us to evaluate these integrals with the result that

$$
H_{m}(t)=2 \pi t\left\{J_{0}(\alpha t) J_{0}\left(M_{1} t\right)+2 \sum_{k=1}^{\infty}(-1)^{k} J_{2 k}(\alpha t) J_{2 k}\left(M_{1} t\right)\right\}
$$


This is of the form described by Gegenbauer's Addition Theorem [6], which for the special case that the function being expanded is a zero th order Bessel function, becomes

$$
J_{0}(p R)=J_{0}(p q) J_{0}(p r)+\sum_{k=1}^{\infty} \cos k \phi J_{k}(p q) J_{k}(p r)
$$

where $R=\left[q^{2}+r^{2}-2 q r \cos \phi\right]^{\frac{1}{2}}$. If we let $p=t, q=\alpha, r=M_{1}$, and $\phi=\frac{\pi}{2}$, we find

$$
R=\left[\alpha^{2}+M_{7}^{2}\right]^{\frac{1}{2}}
$$

Therefore, $H_{m}(t)$ in $(c-14)$ becomes

$$
H_{m}(t)=2 \pi t J_{0}\left[t\left(\alpha^{2}+M_{7}^{2}\right)^{\frac{1}{2}}\right]
$$

If for simplicity, we define the following quantity

$$
h_{m}(\alpha, d)=\frac{M_{1} \sin \frac{m \pi}{2} J_{0}\left(M_{1} g\right)}{k_{m}^{(1)} \sin k_{m}^{(1)} b_{1}} \cdot \frac{\left(\cos k d-\cos k_{m d}^{(1)}\right)}{M_{1}^{2}+\alpha^{2}}
$$

the above results for $I_{4}$ and $I_{5}$ combine to give $Z_{2}$ as

$$
z_{2}=\frac{-i \omega \mu k^{2} I_{0}^{2}}{4 a_{1}^{2} I_{t}^{2}} \int_{-\infty}^{\infty}\left\{\frac{L(\alpha)}{\zeta^{2}} \sum_{m o}, h_{m}(\alpha, d) \sum_{m o} h_{m}(\alpha, d) J_{0}\left[t\left(\alpha^{2}+M_{1}^{2}\right)^{1 / 2}\right]\right\}_{(c-19)} d \alpha
$$

If we now recall that $I_{t}$, the total current at the aperture, is given by $-I_{0} \sin k d, z_{2}$ becomes

$$
z_{2}=\frac{-i \omega \mu k^{2}}{4 a_{1}^{2}} \csc ^{2} k d \int_{-\infty}^{\infty}\left\{\frac{L(\alpha)}{\zeta^{2}} \sum_{m_{0}} h_{m^{\prime}}(\alpha, d) \sum_{m_{0}} h_{m}(\alpha, d) J_{0}\left[t\left(\alpha^{2}+m_{1}^{2}\right)^{\frac{1}{2}}\right]\right\} d \alpha
$$

Unfortunately, the $\alpha$-integration is sufficiently complex that some numerical approach has to be used in evaluating this integral. Notice that for large $m$, since $d<b_{1}, h_{m}(\alpha, d)$ will contain an exponential decay according to $\exp \left(-\alpha\left(b_{1}-d\right)\right)$. Therefore, the integrand will decay rapidly with $\alpha$ and a numerical routine should not prove time consuming. 
This appendix will detail checks of two of the impedance contributions. First, the radiation resistance will be checked against a result given by Tippet [1], and second, $Z_{1}$ will be compared to the rectangular waveguide result given by collin [3]. The radiation resistance $R_{e}^{\prime}$ of an electric dipole inside a TEM cell due to the TEM mode, as given by Tippet [1], is

$$
R_{e}^{\prime}=\frac{2 Z c}{|I|^{2}}\left(d l \frac{I E_{0} \cos \theta 2}{2 V}\right)
$$

where $Z_{c}$ is the characteristic impedance of an RCTL, I is the magnitude of the current, $d l$ is the effective length of the dipole, $E_{0}$ is the TEM mode electric field distribution, $\theta$ is the angle between the dipole direction and the $y$ axis, (for a vertical dipole $\theta=0$ ), and $V$ is the voltage associated with the TEM mode. Because the probe is an electrically short monopole $(k d<1)$ the radiation resistance should be nearly that of a dipole with the same current distribution. Expression (d-1) assumes an elementary dipole, however. We may replace $I E_{0} d l$ with an effective value defined by

$$
\left(I E_{0} d l\right)_{\text {eff }}=\int_{b\{-d}^{b_{1}} I(y) E(y) d y
$$

where $I(y)=\sin k\left(b_{1}-d-y\right)$, and $E(y)$, for a centrally located dipole, is found in [4] to be

$$
E(y)=\frac{2 V}{a} \sum_{m_{0}} \frac{\cosh M\left(b_{1}-y\right)}{\sinh M b_{1}} \sin \frac{m \pi}{2} J_{0}(M g)
$$

Integrating yields the following

$$
\left(I E_{0} d \ell\right)_{\text {eff }}=\frac{2 V k}{a} \sum_{m 0} \frac{(\cos k d-\cosh M d)}{\sinh M b_{1}} \sin \frac{m \pi}{2} J_{0}(m g)
$$

which, if we recall the definition of $h_{m}(\alpha, d)$, may be written

$$
\left(I E_{0} d l\right)_{\text {eff }}=\frac{-2 V k}{a} \sum_{m_{0}} h_{m}(k, d)
$$


If we now normalize by the magnitude of the current, $|I|=\sin k d$, we find $R_{e}^{\prime}$ becomes

$$
R_{e}^{\prime}=\frac{2 z c^{2}}{a^{2}} \csc ^{2} k d\left(\sum_{m o} h_{m}(k, d)\right)^{2}
$$

This may be compared to the radiation resistance $R$ due to the TEM mode given by

$$
R=\frac{2 z c^{2}}{a^{2}} \csc ^{2} k d \sum_{m o} h_{m} \cdot(k, d) \sum_{m o} h_{m}(k, d) J_{0}\left[t\left(k^{2}+m^{2}\right)^{\frac{1}{2}}\right]
$$

The term $h_{m}(k, d)$ has a strong decay in $M$ according to

$$
h_{m}(k, d) \sim \frac{1}{M^{2}} e^{-M\left(b_{1}-d\right)}
$$

Only a few terms will be significant. For these few terms $J_{0}$ will remain near unity since we have assumed that $\pi t / 2 a<1$. Thus, the radiation resistance may be approximated by

$$
R \approx \frac{2 z c^{2}}{a^{2}} \csc ^{2} k d\left(\sum_{m o} h_{m}(k, d)\right)^{2}=R_{e}^{1}
$$

and the results indeed do check.

Next the case of no gap will be considered. As $g \rightarrow 0, L(\alpha) \rightarrow 0$ so only $z_{1}$ contributes. Collin discusses a rectangular waveguide with a short circuit at a distance $\&$ from the probe [3]. Thus we need to modify his result by letting $\ell \rightarrow \infty$. Making the appropriate changes, the radiation resistance for a probe in a rectangular waveguide becomes

$$
R=\frac{\eta_{0}}{2 a b \beta_{10}} \tan ^{2} \frac{k d}{2}
$$

The equivalent reactance is given by

$$
\begin{aligned}
x & =\frac{\eta_{0}}{2 \pi b k} \tan ^{2} \frac{k d}{2}\left\{\ln \left(\frac{4 a}{\pi t}\right)+\frac{k^{2} a^{2}}{\pi^{2}}(.2072)-2\left(1-\frac{t}{a}\right)\right. \\
& \left.-2 k^{2} \sum_{n=1}\left(1-\frac{\sin ^{2} N_{1} d / 2}{\sin ^{2} k d / 2}\right)^{2} \frac{k_{0}\left[t\left(N_{1}^{2}-k^{2}\right)^{\frac{1}{2}}\right]}{N_{1}^{2}-k^{2}}\right\}
\end{aligned}
$$

In order to compare our expression to that of Collin, we must return to Appendix $B(b-4)$ and let $k$ be larger than $M\left(a_{1}=a_{2}=a\right)$. This will create a pole on the real axis when $\alpha=\beta_{10}$. Thus $I_{2}$ becomes 


$$
I_{2}=\frac{-\pi i k^{2}}{2 \beta} g_{10}\left(\bar{x}_{t}, \bar{x}_{t}^{\prime}\right) e^{i \beta} 10|z|+\pi \sum_{m, n=0}^{\prime} \Delta_{n} \frac{k^{2}-N_{1}^{2}}{\Gamma_{m n}^{(1)}} g_{m n}\left(\bar{x}_{t}, \bar{x}_{t}^{\prime}\right) e^{-\Gamma_{m n}^{(1)}|z|}
$$

where the primed summation indicates that the term $m=1, n=0$ is missing. Performing the current integrations yields

$$
\begin{aligned}
& z_{1}=\frac{i \omega \mu I_{0}^{2}}{\pi a b I_{t}^{2}} \int_{0}^{\pi}\left\{\frac{(1-\cos k d)^{2}}{2 i k^{2} \beta_{10}} \cos \left(\frac{\pi t}{2 a} \cos \theta\right) e^{i \beta} 10 t \sin \theta\right.
\end{aligned}
$$

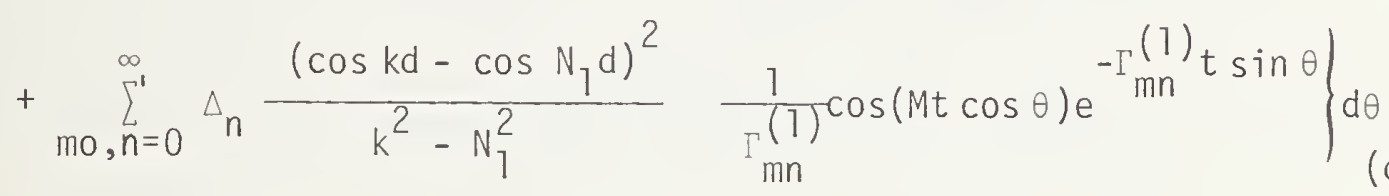

The sum over $m$ and $n$ is precisely that encountered by Collin [3] with the result that

$$
\begin{aligned}
& \sum_{m o, n=0}^{1} \Delta_{n} \frac{\left(\cos k d-\cos N_{1} d\right)}{k^{2}-N_{1}^{2}} \frac{1}{\Gamma_{m n}^{(1)}} \cos (M t \cos \theta) e^{-\Gamma_{m n}^{(1)} t \sin \theta} \\
& =\frac{(1-\cos k d)^{2}}{2 k^{2}} \frac{a}{\pi}\left[\ln \left(\frac{4 a}{\pi t}\right)+\frac{k^{2} a^{2}}{2}(.2072)-2\left(1-\frac{t}{a}\right)\right] \\
& +\frac{a}{\pi} \sum_{n=1}^{\infty} \frac{\left(\cos k d-\cos N_{1} d\right)^{2}}{k^{2}-N_{1}^{2}} k_{0}\left[t\left(N_{1}^{2}-k^{2}\right)^{1 / 2}\right]
\end{aligned}
$$

The significant contribution for $\pi t / 2 a<1$ may be approximated via

$$
\cos \left(\frac{\pi t}{2 a} \cos \theta\right) e^{i \beta} 10 t \sin \theta \approx 1
$$

Combining these results and integrating over $\theta$, which simply introduces a factor of $\pi$, yields

$$
\begin{aligned}
z_{1} \approx & \frac{i \omega \mu I_{0}^{2}}{\pi a b I_{t}^{2}}\left\{\frac{(1-\cos k d)^{2}}{2 i k^{2} \beta_{10}} \pi+\frac{a(1-\cos k d)^{2}}{2 k^{2}}\left[\ln \left(\frac{4 a}{\pi t}\right)+\frac{k^{2} a^{2}}{\pi^{2}}(.2072)\right.\right. \\
& \left.\left.-2\left(1-\frac{t}{a}\right)\right]+a \sum_{n=1}^{\infty} \frac{\left(\cos k d-\cos N_{1} d\right)^{2}}{k^{2}-N_{1}^{2}} k_{0}\left[t\left(N_{1}^{2}-k^{2}\right)^{\frac{1}{2}}\right]\right\}
\end{aligned}
$$


Finally, letting $I_{t}=-I_{0} \sin k d$, and separating $Z_{1}$ into real and imaginary parts $R$ and $X$ respectively, we obtain

$$
\begin{aligned}
R & =\frac{n_{0}}{2 a b k \beta 10} \tan ^{2} \frac{k d}{2} \\
x & =\frac{n_{0}}{2 \pi b k} \tan ^{2} \frac{k d}{2}\left\{\ln \left(\frac{4 a}{\pi t}\right)+\frac{k^{2} a^{2}}{\pi^{2}}(.2072)-2\left(1-\frac{t}{a}\right)\right. \\
& \left.-2 k^{2} \sum_{n=1}^{\infty}\left(1-\frac{\sin ^{2} N_{1} d / 2}{\sin ^{2} k d / 2}\right)^{2} \frac{k_{0}\left[t\left(N_{1}^{2}-k^{2}\right)^{\frac{1}{2}}\right]}{N_{1}^{2}-k^{2}}\right\}
\end{aligned}
$$

which are the precise results found by collin in (d-10) and (d-11). 


\section{APPENDIX E}

\section{SUBROUTINE ZIN}

SUBROUTINE ZIN is used to compute the input impedance $Z$ of a probe inserted into a TEM cell. ZIN calls upon four major subroutines. These are (1) SUBROUTINE ZC which computes the characteristic impedance of a section of RCTL according to (18); (2) SUBROUTINE HMSUM which sums $h_{m}(\alpha, d)$, and $h_{m}(\alpha, d) J_{0}\left[t\left(\alpha^{2}+M_{7}^{2}\right)^{\frac{1}{2}}\right]$; (3) SUBROUTINE XSUM which finds the reactance $X$ given by (20), and (4) SUBROUTINE INTEGR which integrates a complex function over the real axis. INTEGR is called twice in order to evaluate the principal value integral defined by (21). The input to ZIN is mostly via a common block PARAM which contains the cell dimensions $a_{1}, a_{2}, b_{1}, b_{2}$, and $g$, the probe dimensions $d$ and $t$, the constant $\pi$, and the TEM mode propagation constant $k$. Figure 6 gives a flow chart of SUBROUTINE ZIN. The only direct input to ZIN is $\varepsilon$ which sets the desired accuracy. ZIN outputs $Z_{C}, R, X$, and $\delta X$ as well as the total impedance $Z$.

The constant $\varepsilon$ is used to set the upper summation limit in the various sums evaluated by the subroutines. The infinite integral in $\delta x$ is also truncated according to $\varepsilon$. There are three basic sums; the sums inherent in $L(\alpha)$ and $X$, and the summation of the $h_{m}(\alpha, d)$. We will begin with the last. For large $m$, that is $M_{1}^{2}>k^{2}-\alpha^{2}$, we have $K_{m}^{(1)} \sim_{i M_{1}}$, and

$$
h_{m}(\alpha, d) \approx \frac{\sin \frac{m \pi}{2} \cosh M_{1} d}{M_{1}^{2} \sinh M_{1} b_{1}} \approx \frac{\sin \frac{m \pi}{2}}{M_{1}^{2}} e^{-M_{1}\left(b_{1}-d\right)}
$$

We wish to truncate the summation so that the remainder is less than $\varepsilon$. Thus we need to look at the remainder sum

$$
\sum_{m=K}^{\infty} \frac{4 a_{1}^{2}(-1)^{m}}{(2 m-1)^{2} \pi^{2}} e^{-(2 m-1) \pi\left(b_{1}-d\right) / 2 a_{1}}<\varepsilon
$$

Clearly the sum is dominated by the following much simpler sum. 


$$
R_{K}(x)=\sum_{m=K}^{\infty}(-1)^{m} e^{-(2 m-1) x}
$$

so we will set $M$ by requiring that

$$
\frac{4 a_{1}^{2}}{\pi^{2}} R_{K}\left(\frac{\pi\left(b_{1}-d\right)}{2 a_{1}}\right)<\varepsilon
$$

Summing $R_{K}(x)$ is straightforward and yields

$$
R_{K}(x)=\frac{2 e^{-2(K-1) x}}{\cosh x}
$$

Thus inserting this result into $(e-4)$ and solving for $k$ we find

$$
K>\frac{a_{1}}{\pi\left(b_{1}-d\right)} \ln \left[\frac{8 a_{1}^{2}}{\varepsilon \pi \cosh \frac{\pi\left(b_{1}-d\right)}{2 a_{1}}}\right]+1
$$

This condition will be used to truncate the $h_{m}(\alpha, d)$ summation.

Next consider the summation in $L(\alpha)$ given by

$$
\frac{1}{a_{j}} \sum_{m o}\left[\frac{\cot K_{m}^{(j)}}{K_{m}^{(j)}}+\frac{1}{M_{j}}\right]
$$

For large $\mathrm{m}$ the remainder wi1l behave according to

$$
\frac{2}{\pi} \sum_{m=k_{j}}^{\infty} \frac{1}{(2 m-1)}\left(1-\operatorname{coth} \frac{(2 m-1) \pi b_{j}}{2 a_{j}}\right)
$$

Again we choose a simpler dominating sum. For large $m$, such that $(2 m-1) \pi b_{j} / 2 a j>1$, the coth term may be replaced by

$$
\operatorname{coth} \frac{(2 m-1) \pi b_{j}}{2 a_{j}} \approx 1+2 e^{-(2 m-1) \pi b_{j} / a_{j}}
$$

Therefore we need to consider

$$
\frac{4}{\pi} \sum_{m=K_{j}}^{\infty} \frac{1}{2 m-1} e^{-(2 m-1) \pi b_{j} / a_{j}}
$$


Clearly we have

$$
\frac{4}{\pi} \sum_{m=K_{j}}^{\infty} \frac{1}{2 m-1} e^{-(2 m-1) \pi b_{j} / a_{j}}<\frac{4}{\pi} \sum_{m=K_{j}}^{\infty} e^{-(2 m-1) \pi b_{j} / a_{j}}
$$

Summing the R.H.S. and requiring that the result is less than $\varepsilon / 2$ yields

$$
K_{j}>\frac{a_{j}}{2 \pi b_{j}} \text { en }\left[\frac{16}{\varepsilon \pi \sinh \frac{\pi b_{j}}{a_{j}}}\right]+1
$$

This condition will be checked for both $j=1$ and 2 , and the maximum value $k_{j}$ chosen.

We next consider the series contained in $x$. Because both $k d$ and $k t$ are smal1, the decay of the series will be primarily due to the $N_{1}^{2}$ in the denominator. Thus, we will consider the remainder series dominated by

$$
\sum_{n=N}^{\infty} \frac{1}{N_{1}^{2}}, \quad\left(N_{1}=n \pi / b_{1}\right)
$$

This series in turn may be bounded by a simple integral

$$
\sum_{n=N}^{\infty} \frac{1}{N_{1}^{2}}<\left(\frac{b}{\pi}\right)^{2} \int_{N-1}^{\infty} \frac{d x}{x^{2}}=\left(\frac{b}{\pi}\right)^{2} \frac{1}{N-1}
$$

So requiring that the R.H.S. be bounded by $\varepsilon$ implies that

$$
N>\frac{b_{1}^{2}}{\varepsilon \pi}+1
$$

What remains is to bound the truncation of the principal value integral. In terms of $\alpha$ we see $h_{m}(\alpha, d)$ behaving roughtly as $\alpha^{-2}$. Therefore, the total integrand behaves approximately as $\alpha^{-6}$. Thus, the truncation gives a remainder integral similar to

$$
C \int_{x}^{\infty} \alpha^{-6} d \alpha=\frac{c}{5} x^{-5}
$$

where $c=-\omega \mu k^{2} \csc ^{2} k d / 4 a_{1}^{2}$. If we bound this result by $\varepsilon / 2, x$ must satisfy

$$
x>(2 c / 5 \varepsilon)^{1 / 5}
$$


which is the criterion used to set the upper limit of integration.

A listing of the subroutines follows. They were run at the University of Colorado using the MNF compiler, Version 5.4. It should be stressed that $\varepsilon$ sets a percentage error rather than an absolute error. That is $\varepsilon=0.01$ implies $1 \%$ error rather than accuracy to two digits beyond the decimal point. 


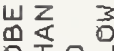

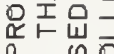

为

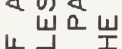

㟔己岂

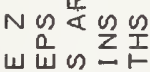

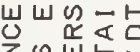

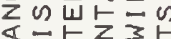

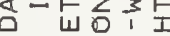

U $z \sum 040$

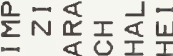

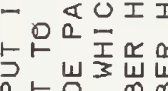

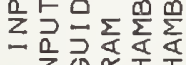

WZ心兄至

Iト岌문

出崖.

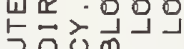

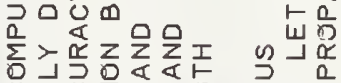

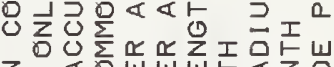

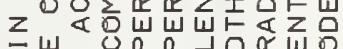

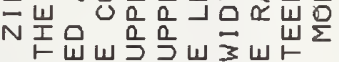

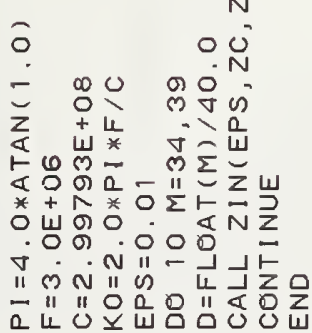

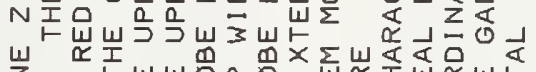

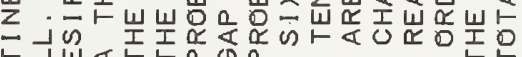

ว

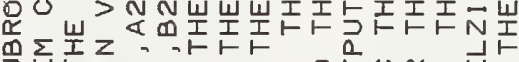

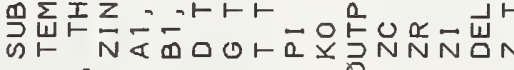

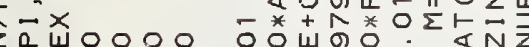
0

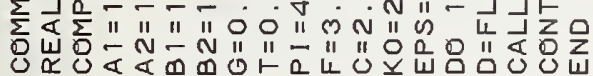

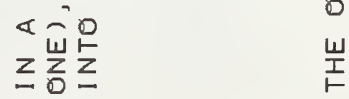

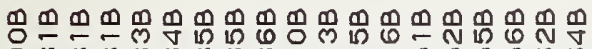

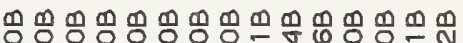

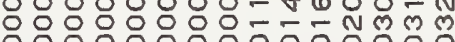
응ㅇㅇㅇㅇㅇㅇㅇㅇㅇㅇㅇㅇㅇㅇ은

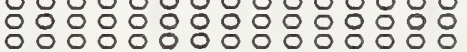

-

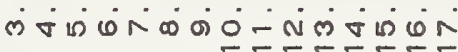




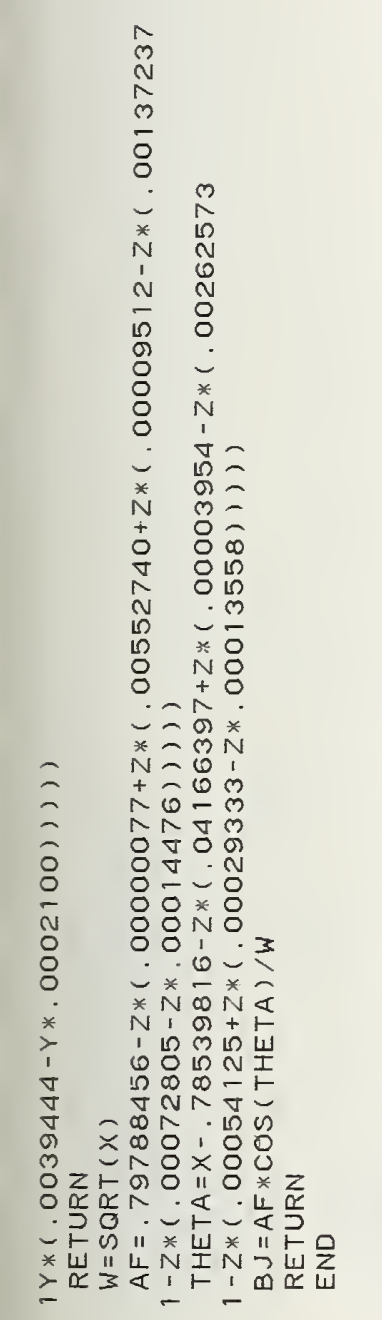

$\underline{-}$

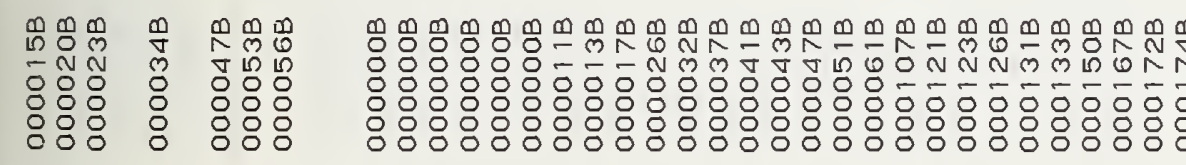

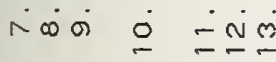

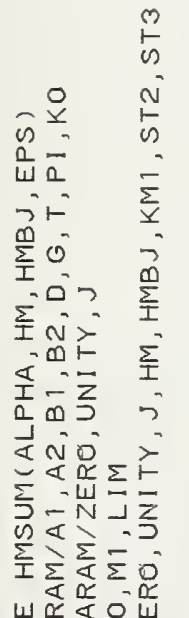

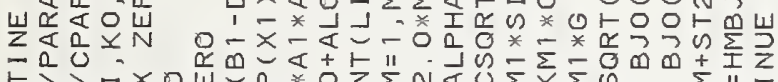

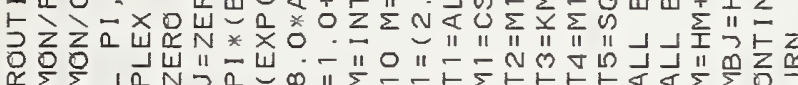

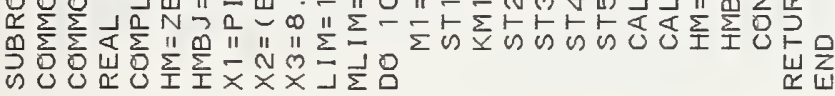

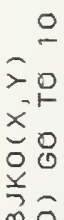

no 1000

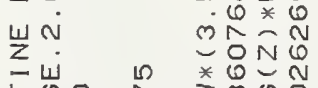

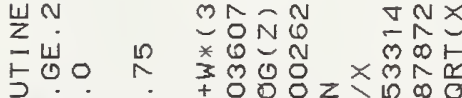
5 .

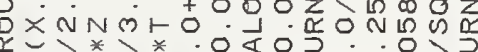

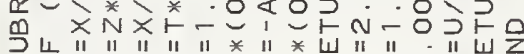

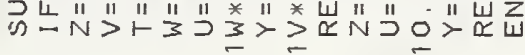
우 $\stackrel{\circ}{-}$

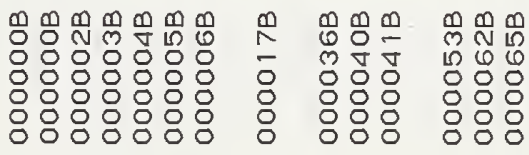

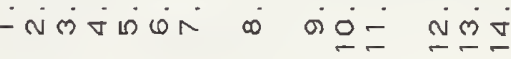



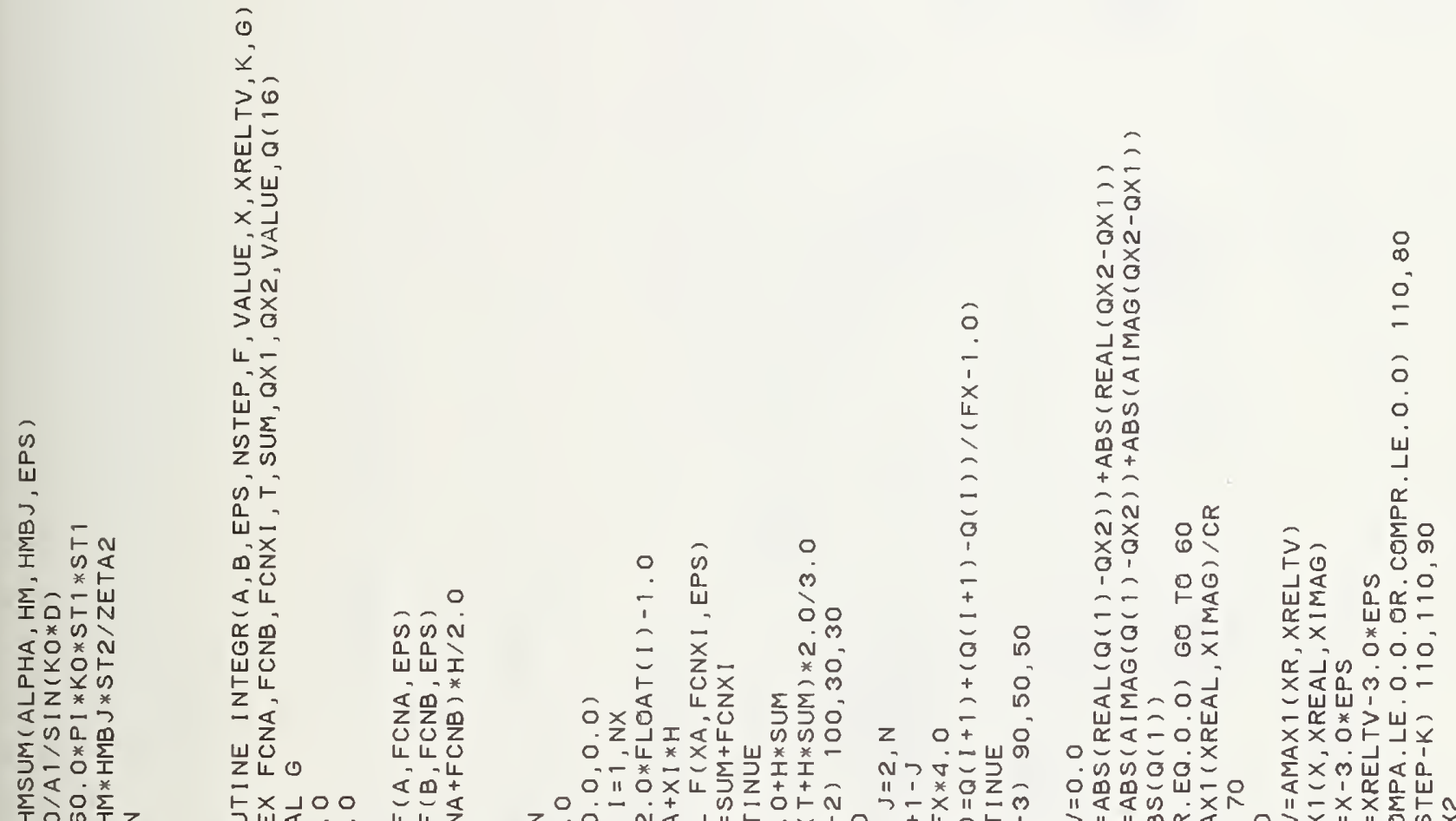

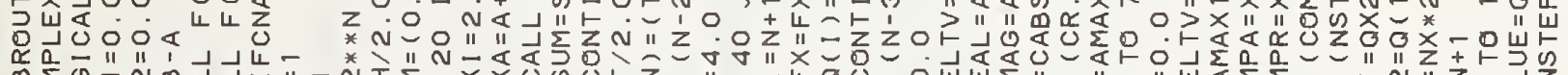

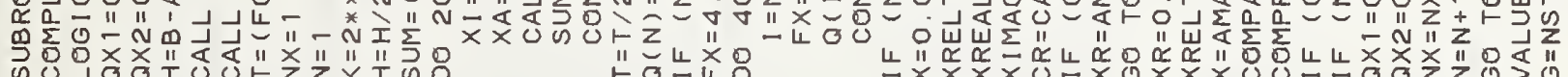

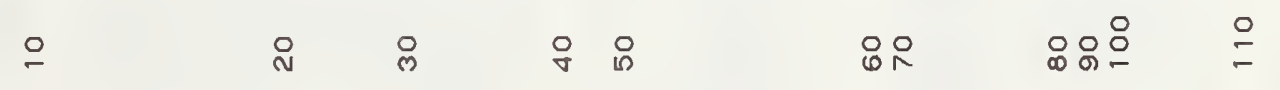

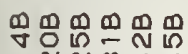

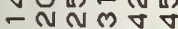
-

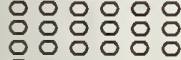

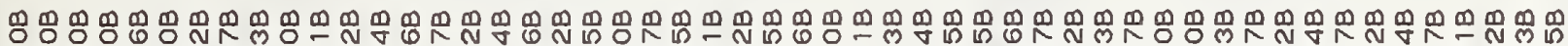

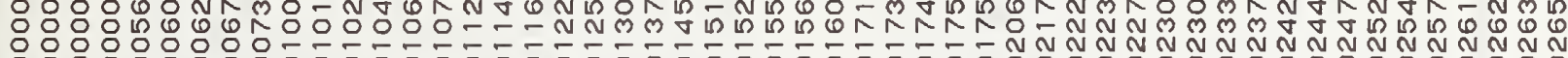

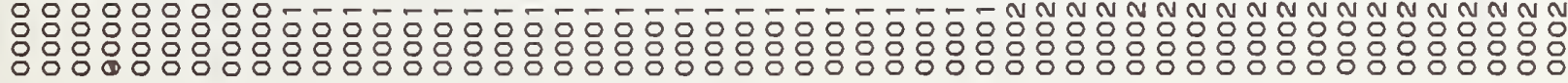

$\therefore \cos =-\frac{N}{2}$

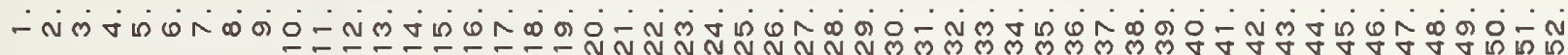


żo

:

N

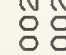

。

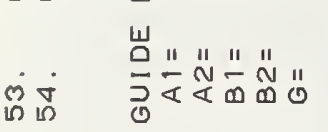

岌

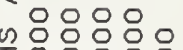

ㄱㅇㅇㅇㅇㅇㅇㅇ

Z - N

$\Sigma$
0
3
0
0
0
4
0
4
4
0

응ㅇㅇㅇㅇㅇㅇ

山 F का

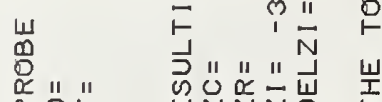

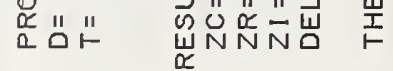

岌岌
3
0
7
0
0
0
0
0
0
1
7
+
0
9
0
0
0
$N$
0
N

0
3
0
1
0
4
0
4

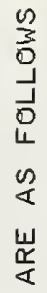

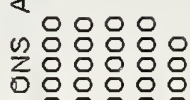

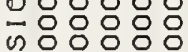
$\sum^{2}$

岸

㟧 " " " " "

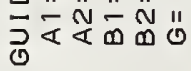

岌 


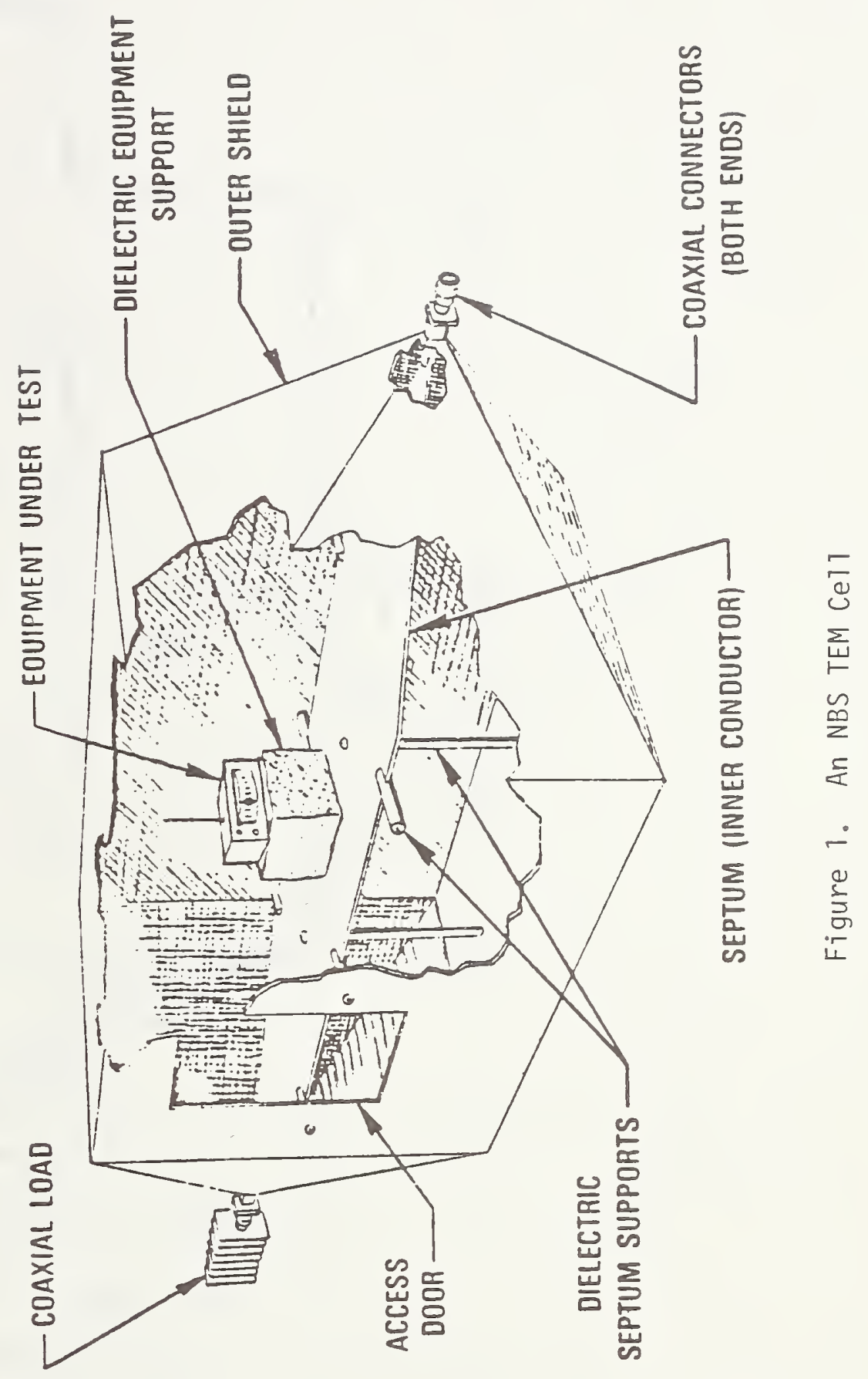




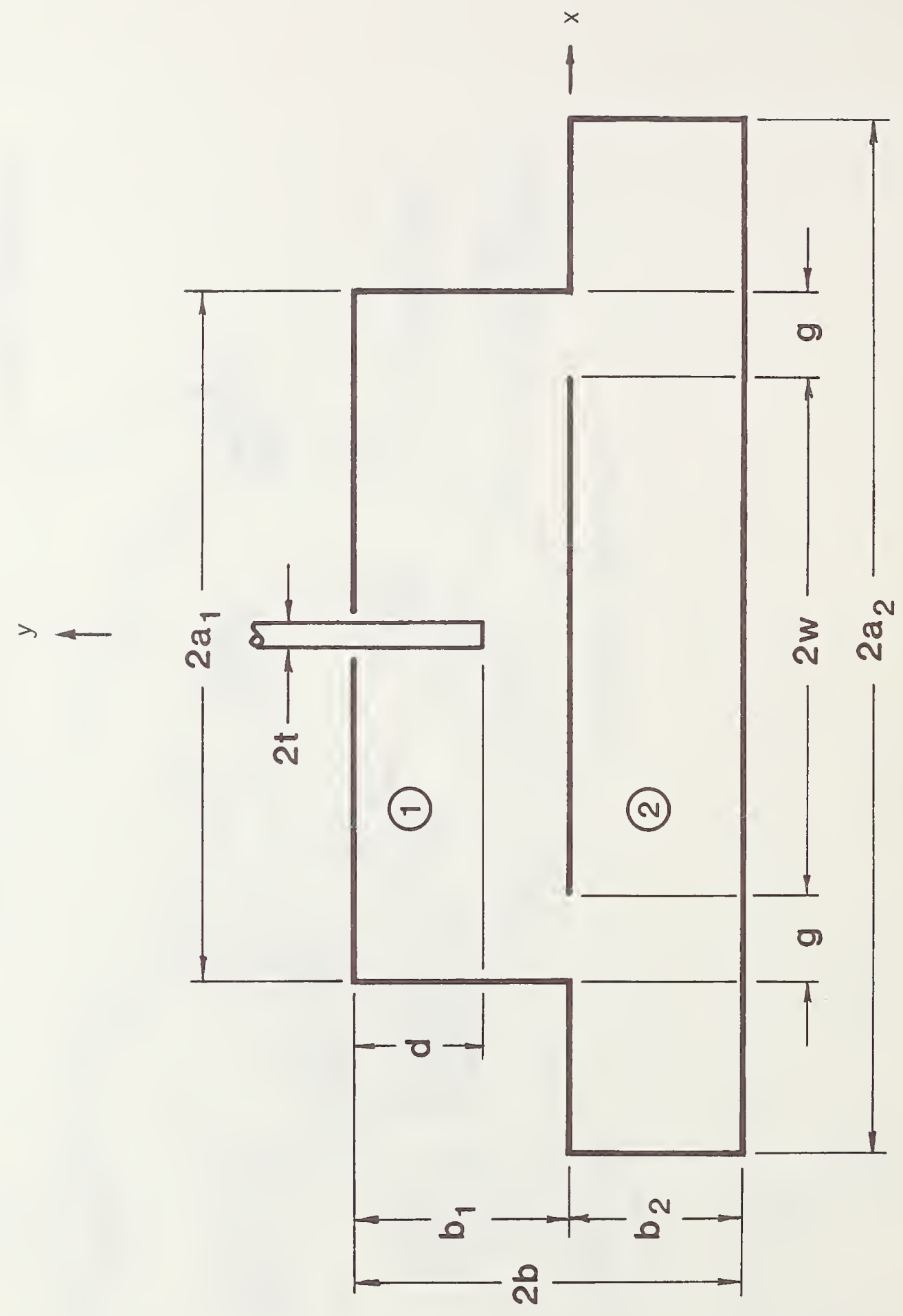

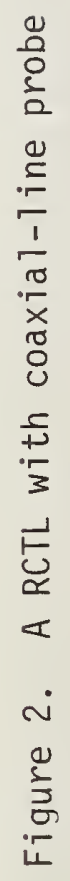




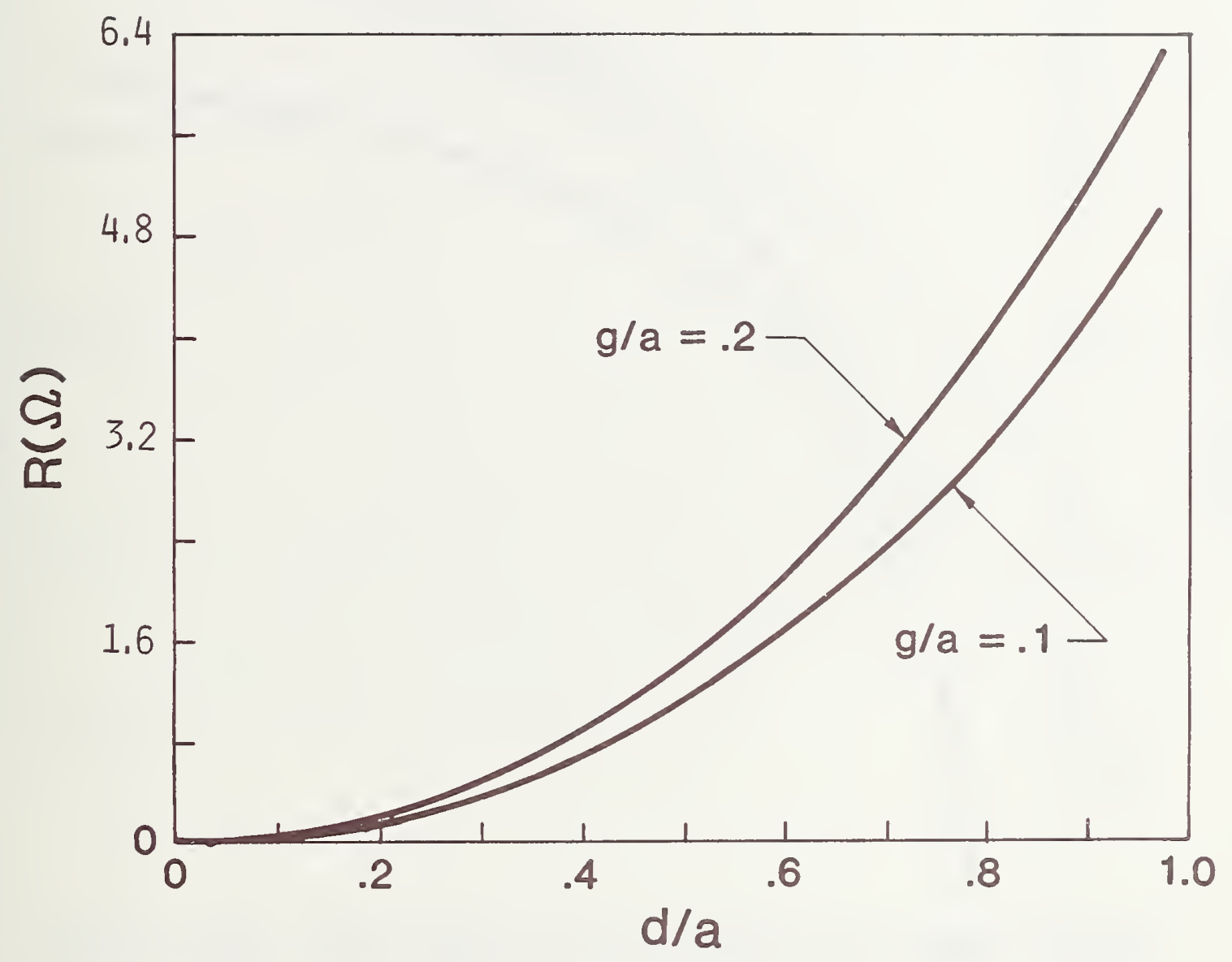

Figure 3. Radiation resistance for $a_{1}=a_{2}=b_{1}=b_{2}=1 \mathrm{~m}$, $t=1 \mathrm{~mm}$, and $\mathrm{f}=1 \mathrm{MHz}$ 


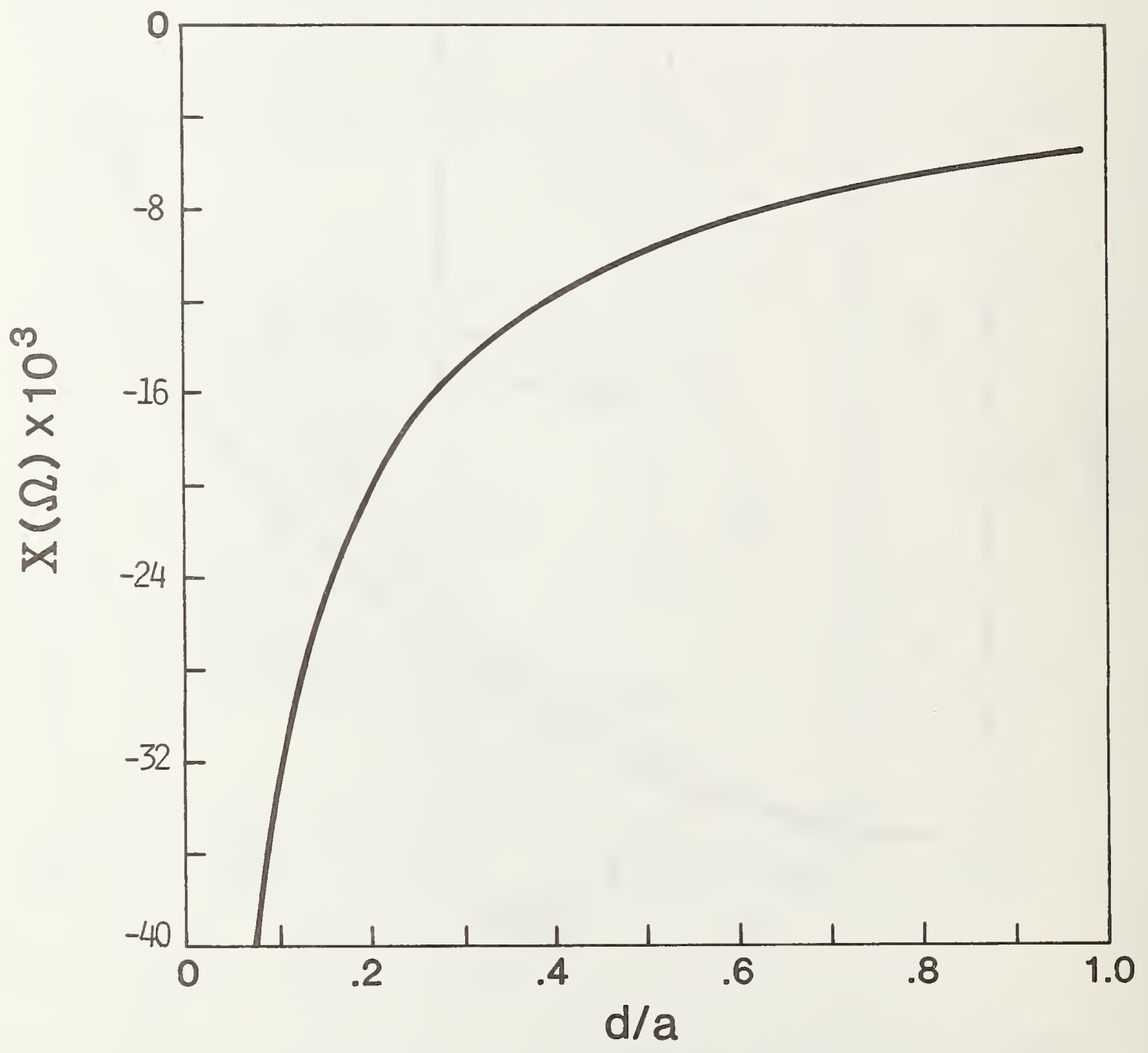

Figure 4. Reactance for $a_{1}=a_{2}=b_{1}=b_{2}=1 \mathrm{~m}, t=1 \mathrm{~mm}$, and $\mathrm{f}=1 \mathrm{MHz}$ 


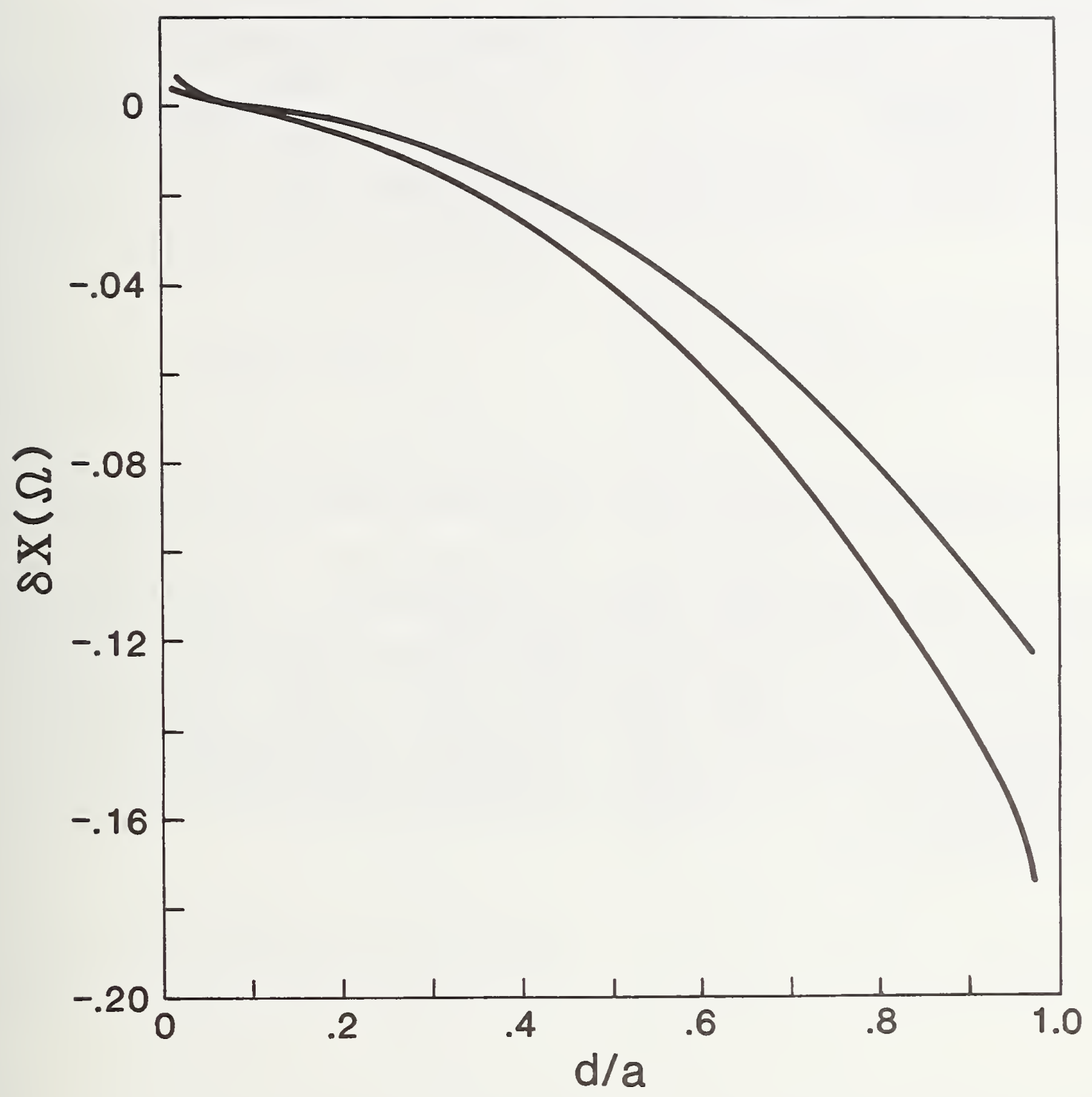

Figure 5. Perturbation reactance for $a_{1}=a_{2}=b_{1}=b_{2}=1 \mathrm{~m}$, $t=1 \mathrm{~mm}$, and $\mathrm{f}=1 \mathrm{MHz}$ 
NBS-114A (REV. 2.8C)

\begin{tabular}{|c|c|c|}
\hline U.S. DEPT. OF COMM. & 1. PUBLICATION OR & 2. Performing Organ. Report No \\
BIBLIOGRAPHIC DATA & REPORT NO. & \\
SHEET (See instructions) & NBS TN-1054 & \\
\hline
\end{tabular}

4. TITL.E AND SUBTITLE

\section{INPUT IMPEDANCE OF A PROBE ANTENNA EXCITING A TEM CELL}

5. $\operatorname{AUTHOR}(S)$

P.F. Wilson, D.C. Chang, and M. T. Ma

6. PERFORMING ORGANIZATION (If joint or other than NBS, see instructions)

NATIONAL BUREAU OF STANDARDS

DEPARTMENT OF COMMERCE

WASHINGTON, D.C. 20234

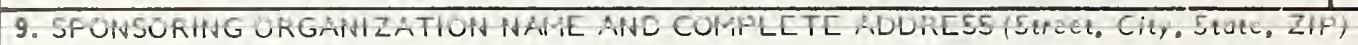

7. Contract/Grant No.

8. Type of Report \& Period Covered

10. SUPPLEMENTARY NOTES

[Document describes a computer program; SF-185, FIPS Software Summary, is attached.

11. ABSTRACT (A 200-word or less factual summary of most significant information. If document includes a significant bibliography or literature survey. mention it here)

The input impedance of a probe antenna exciting a transverse electromagnetic (TEM) transmission Tine cell is formulated by a variational approach. The formulation also utilizes the results from a previous work on the field distribution inside a TEM cell excited by a vertical electrical Hertzian dipole. The final result of impedance is shown to consist of two distinct terms, which are respectively contributed by the ordinary rectangular waveguide and the gap perturbation. Numerical results for both the real and imaginary parts of the impedance are given. The resistive part is found to be proportional to the square of the probe length, and the reactive part largely capacitive.

12. KEY WORDS (Six to twelve entries: alphabetical order: capitalize only proper names; and separate key words by semicolons) Green's function, input impedance; probe antenna; radiation resistance, rectangular coaxial transmission Tine; TEM ce11; variational method.

13. AVAILABILITY

0 Unlimited

For Official Distribution. Do Not Release to NTIS

Order From Superintendent of Documents, U.S. Government Printing Office, Washington, D.C. 20402.

[X Order From National Technical Information Service (NTIS), Springfield, VA. 2216I
14. NO. OF

PRINTED PAGES

52

15. Price

$\$ 9.00$ 


\section{NBS TECHNICAL PUBLICATIONS}

\section{PERIODICALS}

JOURNAL OF RESEARCH-The Journal of Research of the National Bureau of Standards reports NBS research and development in those disciplines of the physical and engineering sciences in which the Bureau is active. These include physics, chemistry, engineering, mathematics, and computer sciences. Papers cover a broad range of subjects, with major emphasis on measurement methodology and the basic technology underlying standardization. Also included from time to time are survey articles on topics closely related to the Bureau's technical and scientific programs. As a special service to subscribers each issue contains complete citations to all recent Bureau publications in both NBS and nonNBS media. Issued six times a year. Annual subscription: domestic $\$ 18$; forcign $\$ 22.50$. Single copy, $\$ 4.25$ domestic; $\$ 5.35$ foreign.

\section{NONPERIODICALS}

Monographs-Major contributions to the technical literature on various subjects related to the Bureau's scientific and technical activities.

Handbooks-Recommended codes of engineering and industrial practice (including safety codes) developed in cosperation with interested industries, professional organizations, and regulatory bodies.

Special Publications-Include proceedings of conferences sponsored by NBS, NBS annual reports, and other special publications appropriate to this grouping such as wall charts, pocket cards, and bibliographies.

Applied Mathematics Series-Mathematical tables, manuals, and studies of special interest to physicists, engineers, chemists, biologists, mathematicians, computer programmers, and others engaged in scientific and technical work.

National Standard Reference Data Series-Provides quantitative data on the physical and chemical properties of materials, compiled from the world's literature and critically evaluated. Developed under a worldwide program coordinated by NBS under the authority of the National Standard Data Act (Public Law 90-396)

NOTE: The principal publication outlet for the foregoing data is the Journal of Physical and Chemical Reference Data (JPCRD) published quarterly for NBS by the American Chemical Society (ACS) and the American Institute of Physics (AIP). Subscriptions, reprints, and supplements available from ACS, 1155 Sixteenth St., NW, Washington, DC 20056
Building Science Series-Disseminates technical information developed at the Bureau on building materials, components, systems, and whole structures. The series presents research results, test methods, and performance criteria related to the structural and environmental functions and the durability and safety characteristics of building elements and systems.

Technical Notes-Studies or reports which are complete in themselves but restrictive in their treatment of a subject. Analogous to monographs but not so comprehensive in scope or definitive in treatment of the subject area. Often serve as a vehicle for final reports of work performed at NBS under the sponsorship of other government agencies.

Voluntary Product Standards-Developed under procedures published by the Department of Commerce in Part 10, Title 15, of the Code of Federal Regulations. The standards establish nationally recognized requirements for products, and provide all concerned interests with a basis for common understanding of the characteristics of the products. NBS administers this program as a supplement to the activities of the private sector standardizing organizations

Consumer Information Series-Practical information, based on NBS research and experience, covering areas of interest to the consumer. Easily understandable language and illustrations provide useful background knowledge for shopping in loday's technological marketplace.

Order the above NBS publications from: Superintendent of Documents, Government Printing Office. Washington, DC 20402

Order the following NBS publications-FIPS and NBSIR's-from the National Technical Information Services, Springfield, VA 22161.

Federal Information Processing Standards Publications (FIPS PUB)-Publications in this series collectively constitute the Federal Information Processing Standards Register. The Register servcs as the official source of information in the Federal Government regarding standards issued by NBS pursuant to the Federal Property and Administrative Services Act of 1949 as amended. Public Law 89-306 (79 Stat. 1127), and as implemented by Executive Order 11717 (38 FR 12315, dated May 11, 1973) and Part 6 of Title 15 CFR (Code of Federal Regulations)

NBS Interagency Reports (NBSIR)-A special series of interim or final reports on work performed by NBS for outside sponsors (both government and non-government). In general, initial distribution is handled by the sponsor: public distribution is by the National Technical Information Services, Springfield, VA 2216I, in paper copy or microfiche form. 
U.5. DEPARTMENT OF COMMERCE

National Bureau of Standards

Washington, O.C. 20234

OFFICIAL BUSINESS

Penalty for Private Use, $\$ 300$
POSTAGE ANO FEES PAIO U.S. OEPARTMENT DF COMMERCE COM-215

\section{U.S.MAIL}

SPECIAL FOURTH-CLASS RATE BOOK 